Preprint typeset in JHEP style - HYPER VERSION

\title{
Bulk Matters on Symmetric and Asymmetric de Sitter Thick Branes
}

\author{
Yu-Xiao Liu ${ }^{1 *}$ Zhen-Hua Zhao ${ }^{2,1}$, Shao-Wen Wei ${ }^{1}$, Yi-Shi Duan ${ }^{1}$ \\ ${ }^{1}$ Institute of Theoretical Physics, Lanzhou University, Lanzhou 730000, P. R. China \\ ${ }^{2}$ Institute of Modern Physics, Chinese Academy of Sciences, Lanzhou 730000, P. R. \\ China \\ E-mail: 1iuyx@lzu.edu.cn, zhaozhenhua@impcas.ac.cn, weishaow06@lzu.cn, \\ ysduan@lzu.edu.cn
}

ABSTRACT: An asymmetric thick domain wall solution with de Sitter $(d S)$ expansion in five dimensions can be constructed from a symmetric one by using a same scalar (kink) with different potentials. In this paper, by presenting the mass-independent potentials of Kaluza-Klein (KK) modes in the corresponding Schrödinger equations, we investigate the localization and mass spectra of various bulk matter fields on the symmetric and asymmetric $d S$ thick branes. For spin 0 scalars and spin 1 vectors, the potentials of KK modes in the corresponding Schrödinger equations are the modified Pöschl-Teller potentials, and there exist a mass gap and a series of continuous spectrum. It is shown that the spectrum of scalar KK modes on the symmetric $d S$ brane contains only one bound mode (the massless mode). However, for the asymmetric $d S$ brane with a large asymmetric factor, there are two bound scalar KK modes: a zero mode and a massive mode. For spin 1 vectors, the spectra of KK modes on both $d S$ branes consist of a bound massless mode and a set of continuous ones, i.e., the asymmetric factor does not change the number of the bound vector KK modes. For spin 1/2 fermions, two types of kink-fermion couplings are investigated in detail. For the usual Yukawa coupling $\eta \bar{\Psi} \phi \Psi$, there exists no mass gap but a continuous gapless spectrum of KK states. For the scalar-fermion coupling $\eta \bar{\Psi} \sin \left(\frac{\phi}{\phi_{0}}\right) \cos ^{-\delta}\left(\frac{\phi}{\phi_{0}}\right) \Psi$ with a positive coupling constant $\eta$, there exist some discrete bound KK modes and a series of continuous ones. The total number of bound states increases with the coupling constant $\eta$. For the case of the symmetric $d S$ brane and positive $\eta$, there are $N_{L}\left(N_{L} \geq 1\right)$ left chiral fermion bound states (including zero mode and massive $\mathrm{KK}$ modes) and $N_{L}-1$ right chiral fermion bound states (including only massive KK modes). For the asymmetric $d S$ brane scenario, the asymmetric factor $a$ reduces the number of the bound fermion KK modes. For large enough $a$, there would not be any right chiral fermion bound mode, but at least one left chiral fermion zero mode.

KEYWORDs: Extra Dimensions, Brane world.

\footnotetext{
${ }^{*}$ Corresponding author.
} 


\section{Contents}

1. Introduction 1

2. Review of the symmetric and asymmetric thick branes 2

3. Localization and mass gaps of various matters on the thick branes

3.1 Spin 0 scalar fields

3.2 Spin 1 vector fields 9

3.3 Spin $1 / 2$ fermion fields 12

3.3 .1 Case I 14

3.3.2 Case II

4. Conclusion and discussion 20

\section{Introduction}

The idea of embedding our universe in a higher dimensional space has received a great of renewed attention. The suggestion that extra dimensions may not be compact [1, 2, 3, [4, [5] or large [6, 7] can provide new insights for solving gauge hierarchy problem [7], i.e., the large difference in magnitude between the Planck and electroweak scales, and the long-standing cosmological constant problem [1, 3, 8]. According to the brane scenarios, gravity is free to propagate in all dimensions, while all the matter fields (electromagnetic, Yang-Mills etc.) are confined to a 3-brane in a high-dimensional space. In Ref. [4], an alternative scenario of the compactification had been proposed. In this scenario, the internal manifold does not need to be compactified to the Planck scale any more, it can be large, or even infinite non-compact, which is one of reasons why this new compactification scenario has attracted so much attention. Among all of the brane world models, there is an interesting and important model in which extra dimensions comprise a compact hyperbolic manifold [9]. The model is known to be free of usual problems that plague the original ADD models and share many common features with Randall-Sundrum (RS) models.

Recently, an increasing interest has been focused on the study of thick brane scenario in higher dimensional space-time 10, 11, 12, 13, 14, 15, 16, since in more realistic models the thickness of the brane should be taken into account. A virtue of these models is that the branes can be obtained naturally rather than introduced by hand. In this scenario the scalar field configuration is usually a kink, which provides a thick brane realization of the brane world as a domain wall in the bulk. However, the inclusion of the gravitational evolution into a dynamic thick wall is a highly non-trivial problem because of the non-linearity of the Einstein equations. For this reason, there are not so many analytic solutions of a dynamic 
thick domain wall. The symmetric de Sitter $(d S)$ branes have been studied in five and higher dimensional spacetimes, for examples in [14, 17, 18]. Ref. 19] presented a method to construct asymmetric thick $d S$ brane solutions from known ones, where the spacetimes associated to them are physically different. With the method, asymmetric brane worlds with $d S$ expansion were obtained. These branes interpolate between two spacetimes with different cosmological constants, and the vacua correspond to $d S$ and $A d S$ geometry. It was shown that gravity is localized on such branes.

In brane world scenarios, an important and complex question is localization of various bulk fields on a brane by a natural mechanism. It is well known that massless scalar fields [20] and graviton [4] can be localized on branes of different types. However, spin 1 Abelian vector fields can not be localized on the RS brane in five dimensions, but can be localized on the RS brane in some higher-dimensional cases [21] or on the thick $d S$ brane and Weyl thick brane [22]. The localization problem of spin $1 / 2$ fermions on thick branes is interesting and important. Fermions do not have normalizable zero modes in five and six dimensions without the scalar-fermion coupling [20, 21, 22, 23, 24, 25, 26, 27, 28, 29, 30, 31, 32]. In Ref. [30], the authors obtained trapped discrete massive fermion states on the brane, which in fact are quasi-bound and have a finite probability of escaping into the bulk. In fact, fermions can escape into the bulk by tunnelling, and the rate depends on the parameters of the scalar potential [31]. In five dimensions, with the scalar-fermion coupling, there may exist a single bound state and a continuous gapless spectrum of massive fermion KaluzaKlein (KK) states [22, 33]. While in some other brane models, there exist finite discrete KK states (mass gap) and a continuous gapless spectrum starting at a positive $m^{2}$ [34, 35].

Since a physically different asymmetric thick $d S$ brane solution can be constructed from a known symmetric one by including an asymmetric factor, we will address the localization and mass spectrum problems of various bulk matters on the symmetric and asymmetric $d S$ branes, and investigate the influence of the asymmetric factor on the mass spectra of bulk matters in this paper. We will show that all bulk matters (scalars, vectors and fermions) can be localized on these branes and the corresponding mass spectra have a mass gap (for spin $1 / 2$ fermions the scalar-fermion coupling should not be the usual Yukawa coupling $\eta \bar{\Psi} \phi \Psi$ in order to trap the zero mode). The large asymmetric factor increases the number of the scalar bound states but reduces that of the fermion ones, and does not change the number of the vector bound states.

The organization of the paper is as follows: In section 2, we first review the symmetric and asymmetric $d S$ thick branes in 5-dimensional space-time. Then, in section 3 , we study the localization and mass spectra of various bulk fields on the symmetric and asymmetric

thick branes by presenting the shapes of the potentials of the corresponding Schrödinger problem. For spin 1/2 fermions, we consider two different types of scalar-fermion interactions. Finally, the conclusion and summary are given.

\section{Review of the symmetric and asymmetric thick branes}

Let us consider thick branes arising from a real scalar field $\phi$ with a scalar potential $V(\phi)$. 
The action for such a system is given by

$$
S=\int d^{5} x \sqrt{-g}\left[\frac{1}{2 \kappa_{5}^{2}} R-\frac{1}{2} g^{M N} \partial_{M} \phi \partial_{N} \phi-V(\phi)\right],
$$

where $R$ is the scalar curvature and $\kappa_{5}^{2}=8 \pi G_{5}$ with $G_{5}$ the 5 -dimensional Newton constant. Here we set $\kappa_{5}=1$. The line-element for a 5 -dimensional spacetime with planar-paralell symmetry is assumed as

$$
\begin{aligned}
d s^{2} & =\mathrm{e}^{2 A(z)}\left(\hat{g}_{\mu \nu}(x) d x^{\mu} d x^{\nu}+d z^{2}\right) \\
& =\mathrm{e}^{2 A(z)}\left(-d t^{2}+e^{2 \beta t} d x^{i} d x^{i}+d z^{2}\right),
\end{aligned}
$$

where $\mathrm{e}^{2 A(z)}$ is the warp factor and $z$ stands for the extra coordinate. For the positive constant $\beta>0$, we will have dynamic solutions. The scalar field is considered to be a function of $z$ only, i.e., $\phi=\phi(z)$. In the model, the potential could provide a realization of a thick brane, and the soliton configuration of the scalar field dynamically generate the domain wall configuration with warped geometry. The field equations generated from the action (2.1) with the ansatz (2.2) reduce to the following coupled nonlinear differential equations

$$
\begin{aligned}
\phi^{2} & =3\left(A^{2}-A^{\prime \prime}-\beta^{2}\right), \\
V(\phi) & =\frac{3}{2} e^{-2 A}\left(-3 A^{\prime 2}-A^{\prime \prime}+3 \beta^{2}\right), \\
\frac{d V(\phi)}{d \phi} & =e^{-2 A}\left(3 A^{\prime} \phi^{\prime}+\phi^{\prime \prime}\right),
\end{aligned}
$$

where the prime denotes derivative with respect to $z$. For positive and vanishing $\beta$ we will obtain dynamic and static solutions, respectively.

A symmetric thick domain wall with $d S$ expansion in five dimensions for the potential

$$
V(\phi)=\frac{1+3 \delta}{2 \delta} 3 \beta^{2}\left(\cos \frac{\phi}{\phi_{0}}\right)^{2(1-\delta)},
$$

was found in Refs. [36, 37]:

$$
\begin{aligned}
e^{2 A} & =\cosh ^{-2 \delta}\left(\frac{\beta z}{\delta}\right), \\
\phi & =\phi_{0} \arctan \left(\sinh \frac{\beta z}{\delta}\right),
\end{aligned}
$$

where $\phi_{0}=\sqrt{3 \delta(1-\delta)}, 0<\delta<1$ and $\beta>0$. In this system, The scalar field takes values $\pm \phi_{0} \pi / 2$ at $z \rightarrow \pm \infty$, corresponding to two consecutive minima of the potential with cosmological constant $\Lambda=0$. The scalar configuration in fact is a kink, which provides a thick brane realization of the brane world as a domain wall in the bulk. $\delta$ plays the role of the wall's thickness. The thick brane has a well-defined distributional thin wall limit when $\delta \rightarrow 0$ [38] and can localize gravity on the wall [14]. Note that for the cases where $1 / 2<\delta<1$, the hypersurfaces $|z|=\infty$ represent non-scalar spacetime singularities [14]. 
An asymmetric thick domain wall solution with $d S$ expansion in five dimensions for the same kink configuration $\phi$ in (2.8) was found in Ref. [19]:

$$
\begin{aligned}
e^{-A}= & \cosh ^{\delta}\left(\frac{\beta z}{\delta}\right)+\frac{i a \delta}{\beta-2 \beta \delta} \cosh ^{-\delta}\left(\frac{\beta z}{\delta}\right) \operatorname{coth}\left(\frac{\beta z}{\delta}\right) \\
& \times\left|\sinh \left(\frac{\beta z}{\delta}\right)\right|{ }_{2} F_{1}\left(\frac{1}{2}-\delta, \frac{1}{2}, \frac{3}{2}-\delta, \cosh ^{2}\left(\frac{\beta z}{\delta}\right)\right),
\end{aligned}
$$

where ${ }_{2} F_{1}$ is the hypergeometric function. Here we will consider the case $\delta=1 / 2$ for convenience:

$$
\begin{aligned}
e^{2 A}= & \frac{\beta^{2} \operatorname{sech} 2 \beta z}{[\beta+a \arctan (\tanh \beta z)]^{2}}, \\
\phi= & \frac{\sqrt{3}}{2} \arctan (\sinh 2 \beta z), \\
V(\phi)= & \frac{3}{2}\left|\cos \frac{2 \phi}{\sqrt{3}}\right|\left\{-4 a^{2}+5 \beta^{2}-8 a \beta \tan \frac{2 \phi}{\sqrt{3}}\right. \\
& +5 a^{2} \arctan ^{2}\left[\tanh \left(\frac{1}{2} \operatorname{arcsinh}\left(\tan \frac{2 \phi}{\sqrt{3}}\right)\right)\right] \\
& \left.+2 a \arctan \left[\tanh \left(\frac{1}{2} \operatorname{arcsinh}\left(\tan \frac{2 \phi}{\sqrt{3}}\right)\right)\right]\left(5 \beta-4 a \tan \frac{2 \phi}{\sqrt{3}}\right)\right\},
\end{aligned}
$$

where $|a|<4 \beta / \pi$ in order to prevent singularities in the metric tensor. The parameter $a$ decides the asymmetry of the solution. For $a=0$, we recover the symmetric domain wall solution. For positive (negative) $a$, the spacetime for $z \rightarrow+\infty$ is asymptotically $A d S(d S)$ with cosmological constant $-3 a(4 \beta+a \pi)$ and for $z \rightarrow-\infty$ is asymptotically $d S$ (AdS) with cosmological constant $3 a(4 \beta-a \pi)$. The scalar curvature $R$ and the energy density $\rho$ for the $d S$ brane are calculated as follows:

$$
\begin{aligned}
R= & 4 \operatorname{sech} 2 \beta z\left[7 \beta^{2}-5 a^{2}+14 a \beta \arctan (\tanh \beta z)\right. \\
& \left.+7 a^{2} \arctan ^{2}(\tanh \beta z)-10 a(\beta+a \arctan (\tanh \beta z)) \sinh 2 \beta z\right], \\
\rho= & \operatorname{sech} 2 \beta z\left[3 \beta^{2}-2 a^{2}+6 a \beta \arctan (\tanh \beta z)\right. \\
& \left.+3 a^{2} \arctan ^{2}(\tanh \beta z)-4 a(\beta+a \arctan (\tanh \beta z)) \sinh 2 \beta z\right] .
\end{aligned}
$$

The shapes for the metric factor $e^{2 A}$, the potential $V(\phi)$, the scalar curvature $R$, and the density energy $\rho$ are shown in Fig. 1.

\section{Localization and mass gaps of various matters on the thick branes}

In this section let us investigate whether various bulk mater fields such as spin 0 scalars, spin 1 vectors and spin 1/2 fermions can be localized on the thick branes by means of only the gravitational interaction. Of course, we have implicitly assumed that various bulk fields considered below make little contribution to the bulk energy so that the solutions given in previous section remain valid even in the presence of bulk fields. We will analyze the spectra of various mater fields for the thick brane by presenting the potential of the 

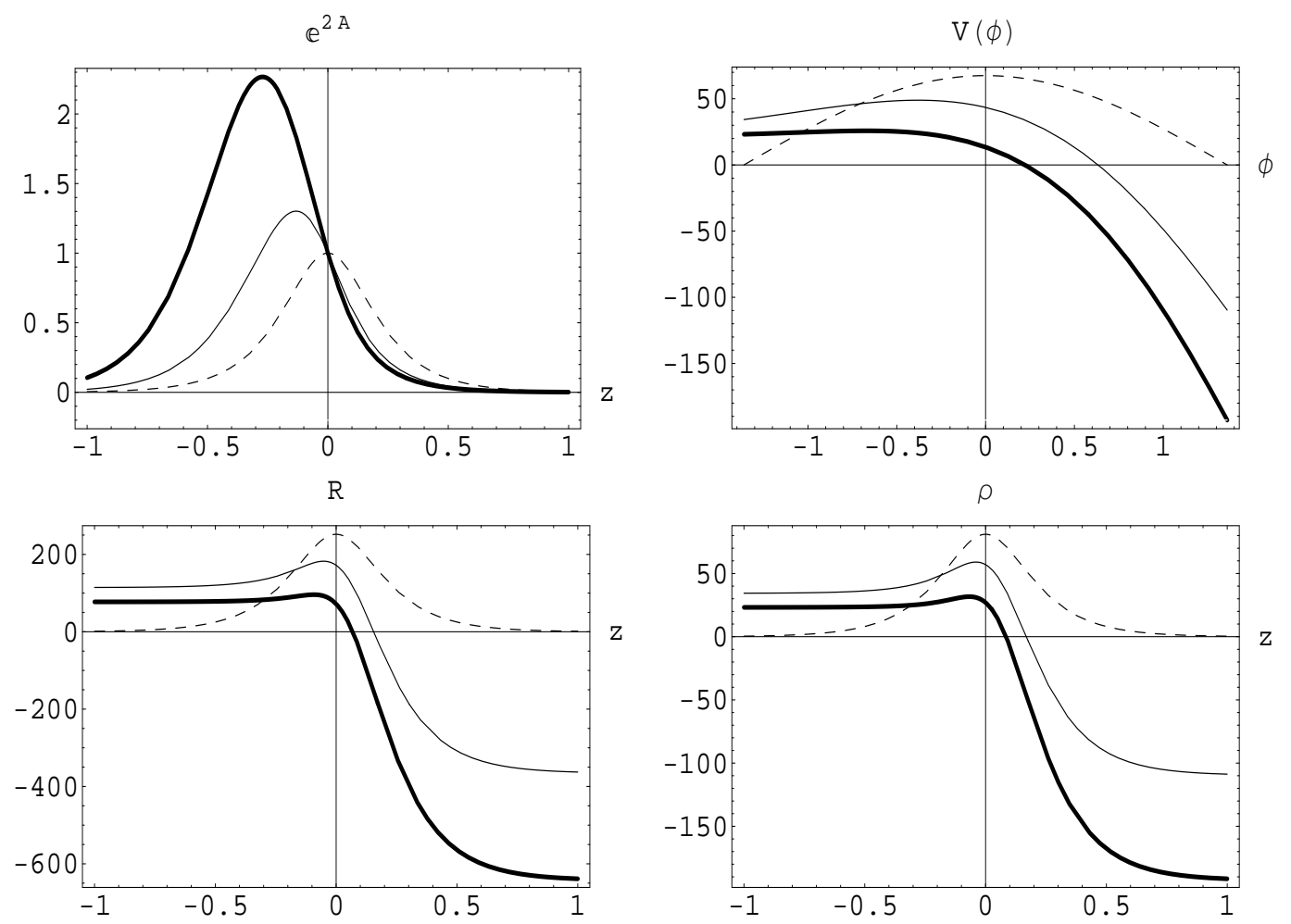

Figure 1: The shapes of the metric factor $e^{2 A}$, scalar potential $V(\phi)$, the scalar curvature $R$, and the energy density $\rho$ for the $d S$ branes with $\beta=3$. The parameter $a$ is set to $a=0$ for dashed lines, $a=2$ for thin lines, and $a=3$ for thick lines.

corresponding Schrödinger equation. It can be seen from the following calculations that the mass-independent potential can be obtained conveniently with the conformally flat metric (2.2).

\subsection{Spin 0 scalar fields}

We first study localization of a real scalar field on the branes obtained in previous section. Let us start by considering the action of a massless real scalar coupled to gravity

$$
S_{0}=-\frac{1}{2} \int d^{5} x \sqrt{-g} g^{M N} \partial_{M} \Phi \partial_{N} \Phi .
$$

By considering the conformally flat metric (2.2) the equation of motion derived from (3.1) is read

$$
\frac{1}{\sqrt{-\hat{g}}} \partial_{\mu}\left(\sqrt{-\hat{g}} \hat{g}^{\mu \nu} \partial_{\nu} \Phi\right)+e^{-3 A} \partial_{z}\left(e^{3 A} \partial_{z} \Phi\right)=0
$$

Then, by decomposing $\Phi(x, z)=\sum_{n} \phi_{n}(x) \chi_{n}(z) e^{3 A / 2}$ and demanding $\phi_{n}(x)$ satisfies the 4-dimensional massive Klein-Gordon equation $\left(\frac{1}{\sqrt{-\hat{g}}} \partial_{\mu}\left(\sqrt{-\hat{g}} \hat{g}^{\mu \nu} \partial_{\nu}\right)-\mu_{n}^{2}\right) \phi_{n}(x)=0$, we obtain the equation for $\chi_{n}(z)$ :

$$
\left[-\partial_{z}^{2}+V_{0}(z)\right] \chi_{n}(z)=\mu_{n}^{2} \chi_{n}(z)
$$


which is a Schrödinger equation with the effective potential given by

$$
V_{0}(z)=\frac{3}{2} A^{\prime \prime}+\frac{9}{4} A^{\prime 2},
$$

where $\mu_{n}$ is the mass of the KK excitations. It is clear that $V_{0}(z)$ defined in (3.4) is a mass-independent potential.

The full 5-dimensional action (3.1) reduces to the standard 4-dimensional action for the massive scalars

$$
S_{0}=-\frac{1}{2} \sum_{n} \int d^{4} x \sqrt{-\hat{g}}\left(\hat{g}^{\mu \nu} \partial_{\mu} \phi_{n} \partial_{\nu} \phi_{n}+\mu_{n}^{2} \phi_{n}^{2}\right)
$$

when integrated over the extra dimension, in which it is required that Eq. (3.3) is satisfied and the following orthonormality condition is obeyed:

$$
\int_{-\infty}^{\infty} d z \chi_{m}(z) \chi_{n}(z)=\delta_{m n}
$$

For the symmetric and asymmetric $d S$ brane world solutions (2.7) and (2.10), the potentials corresponding to (3.4) are

$$
V_{0}^{S}(z)=\frac{3 \beta^{2}}{4 \delta}\left(3 \delta-(2+3 \delta) \operatorname{sech}^{2}(\beta z / \delta)\right)
$$

and

$$
\begin{aligned}
V_{0}^{A}(z)= & \frac{9 \beta^{2}}{4}+\frac{15 a \beta^{2} \operatorname{sech} 2 \beta z \tanh 2 \beta z}{2(\beta+a \arctan \tanh \beta z)} \\
& -\frac{3 \beta^{2}\left(7 \beta^{2}-5 a^{2}+7 a \arctan \tanh \beta z(2 \beta+a \arctan \tanh \beta z)\right)}{4(\beta+a \arctan \tanh \beta z)^{2} \cosh ^{2}(2 \beta z)}, \quad\left(\delta=\frac{1}{2}\right)
\end{aligned}
$$

respectively. For the case $a=0$, the potential (3.8) is reduced to (3.7) with $\delta=1 / 2$ :

$$
V_{0}^{S}(z)=\frac{3}{4} \beta^{2}\left(3-7 \operatorname{sech}^{2}(2 \beta z)\right) \cdot\left(\delta=\frac{1}{2}\right)
$$

We first investigate the potential (3.7) for the symmetric $d S$ brane. It has a minimum (negative value) $-\frac{3 \beta^{2}}{2 \delta}$ at $z=0$ and a maximum (positive value) $\frac{9}{4} \beta^{2}$ at $z= \pm \infty$. Let $p=\beta / \delta$ and $q=1+3 \delta / 2$, Eq. (3.3) with the potential (3.7) turns into the well-known Schrödinger equation with $E_{n}=\mu_{n}^{2}-\frac{9}{4} \delta^{2} p^{2}$ :

$$
\left[-\partial_{z}^{2}-q(q-1) p^{2} \operatorname{sech}^{2}(p z)\right] \chi_{n}=E_{n} \chi_{n} .
$$

For this equation with a modified Pöschl-Teller potential, the energy spectrum of bound states is found to be

$$
E_{n}=-p^{2}(q-1-n)^{2}
$$

or

$$
\mu_{n}^{2}=n(3 \delta-n) \frac{\beta^{2}}{\delta^{2}}
$$




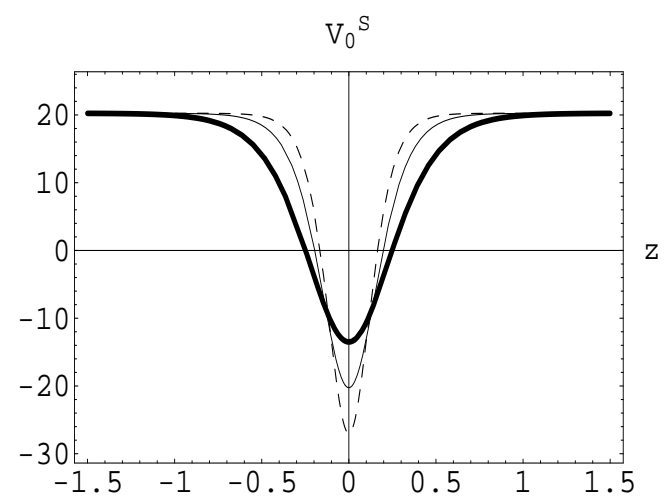

Figure 2: The shapes of the potential $V_{0}^{S}(z)$ for the symmetric $d S$ brane. The parameters are set to $\beta=3, \delta=1 / 2$ for dashed line, $\delta=2 / 3$ for thin line, and $\delta \rightarrow 1$ for thick line.

where $n$ is an integer and satisfies $0 \leq n<\frac{3}{2} \delta$. It is clear that the energy for $n=0$ or $\mu_{0}=0$ always belongs to the spectrum of the potential (3.7) for $\delta>0$. For $0<\delta \leq \frac{2}{3}$, there is only one bound state, i.e., the ground state

$$
\chi_{0}(z)=\sqrt{\frac{\beta \Gamma\left(\frac{1}{2}+\frac{3 \delta}{2}\right)}{\delta \sqrt{\pi} \Gamma\left(\frac{3 \delta}{2}\right)}} \operatorname{sech}^{3 \delta / 2}(\beta z / \delta)
$$

with $\mu_{0}=0$, which is just the normalized zero-mass mode and also shows that there is no tachyonic scalar mode. The continuous spectrum starts with $\mu^{2}=\frac{9}{4} \beta^{2}$ and asymptotically turn into plane waves, which represent delocalized KK massive scalars. For $\frac{2}{3}<\delta<1$, there are two bound states, one is the ground state (3.13), another is the first exited state

$$
\chi_{1}(z) \propto \operatorname{sech}^{3 \delta / 2}(\beta z / \delta) \sinh z
$$

with mass $\mu_{1}^{2}=(3 \delta-1) \beta^{2} / \delta^{2}$. The continuous spectrum also start with $\mu^{2}=\frac{9}{4} \beta^{2}$. From above analysis, we come to the conclusion: for $0<\delta \leq \frac{1}{2}$, there is only one bound state (is massless mode) for the symmetric potential (3.7).

Next we turn to the potential (3.8) for asymmetric $d S$ brane. It has a negative value at some $z_{0}\left(z_{0}<0\right.$ for $a>0$ and $z_{0}>0$ for $\left.a<0\right)$ and the asymptotic behavior: $V_{0}^{A}(z= \pm \infty)=\frac{9}{4} \beta^{2}$, which implies that there is also a mass gap. For the massless mode $\chi_{0}(z)$ with $\mu^{2}=0$, the Schrödinger equation (3.3) with the potential (3.8) can be solved analytically, and the normalizable eigenfunction is found to be

$$
\chi_{0}(z) \propto\left(\frac{\beta^{2} \operatorname{sech} 2 \beta z}{[\beta+a \arctan (\tanh \beta z)]^{2}}\right)^{3 / 4} .
$$

This zero mode is the ground state since it has no node. For the limit $a \rightarrow 0$, the massless mode (3.15) is reduced to (3.13) but with $\delta=1 / 2$. Now, we ask an interesting question: are there other bound states except the zero mode for the asymmetric potential (3.8)? This is very important for producing 4-dimensional massive scalars. If the answer is yes, we will get massive scalars on the asymmetric $d S$ brane. We have known that there 


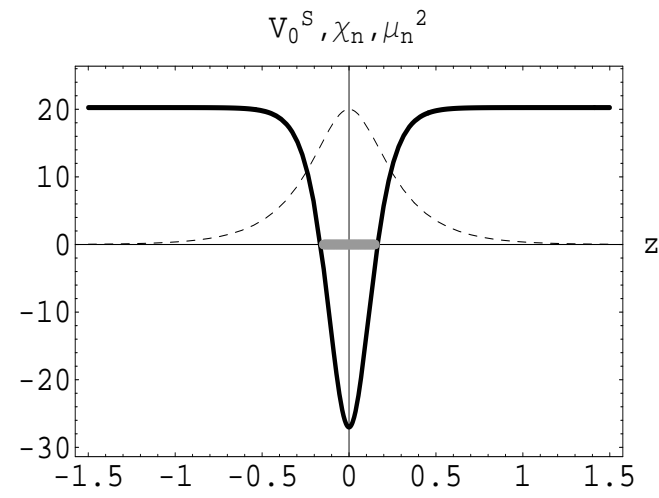

(a) $\delta=1 / 2$

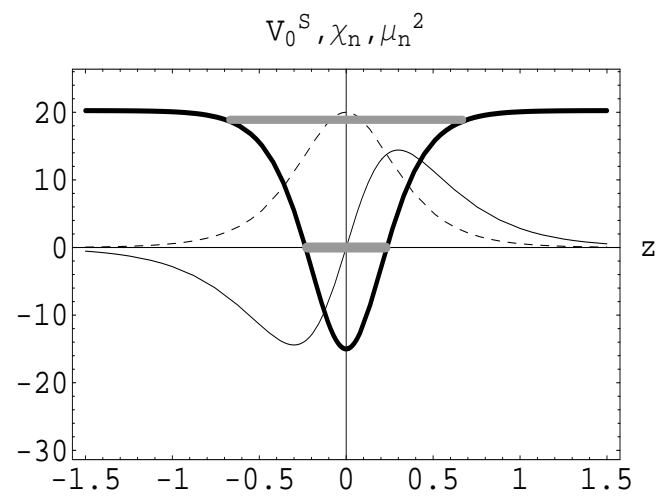

(b) $\delta=0.9$

Figure 3: The shapes of the potential $V_{0}^{S}(z)$ (thick lines), KK modes $\chi_{n}(z)$ (dashed lines for $\chi_{0}(z)$ and thin lines for $\left.\chi_{1}(z)\right)$ and the mass spectrum (thick gray lines) for symmetric $d S$ brane with $\beta=3, \delta=1 / 2$ and $\delta=0.9$.

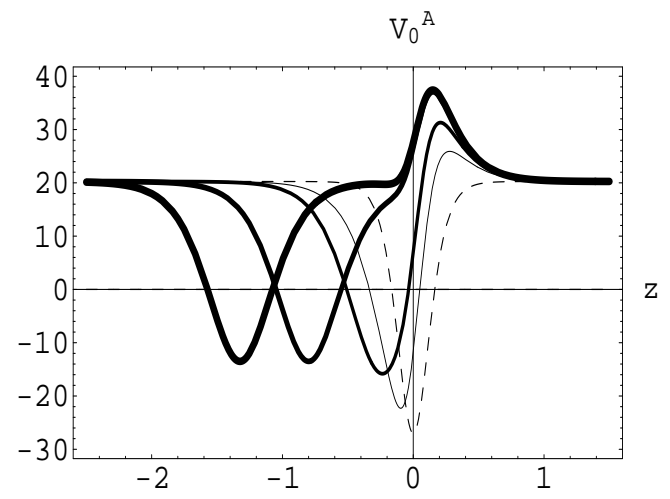

Figure 4: The shapes of the potential $V_{0}^{A}(z)$ for the asymmetric $d S$ brane. The parameters are set to $\beta=3, \delta=1 / 2, a=0$ for dashed line, and $a=2,3,3.78,3.818$ for solid lines with thickness increases with $a$.

is no any massive bound state for the symmetric potential (3.9), the limit case of the current asymmetric one. Hence we can extrapolate that the answer should be no for small asymmetric factor $a$. However, what will happen for large $a$ ? We note that the asymmetric potential (3.8) has the same asymptotic behavior as the symmetric case: $V_{0}^{A}( \pm \infty)=\frac{9}{4} \beta^{2}$, but a different minimum $V_{0 \text { min }}^{A}$, which is larger than that of the symmetric potential. The absolute value of the minimum of the asymmetric potential decreases with the increase of the asymmetry (see Fig. 1 ). This leads to the increase of the relative depth of the potential well $V_{0}^{A}( \pm \infty) /\left|V_{0 \text { min }}^{A}\right|$, which indicates that the potential well may trap more bound stats. By numerical method, we do get a massive bound state with $\mu_{1}^{2}=18.11$ at $a=3.818$ (see Fig. 5). 


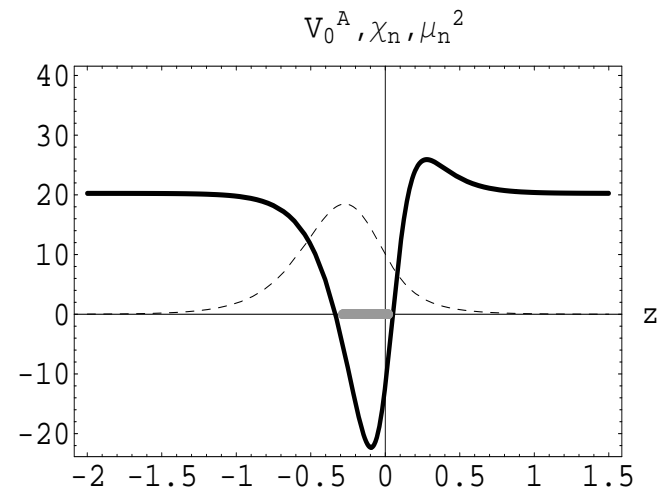

(a) $a=2$

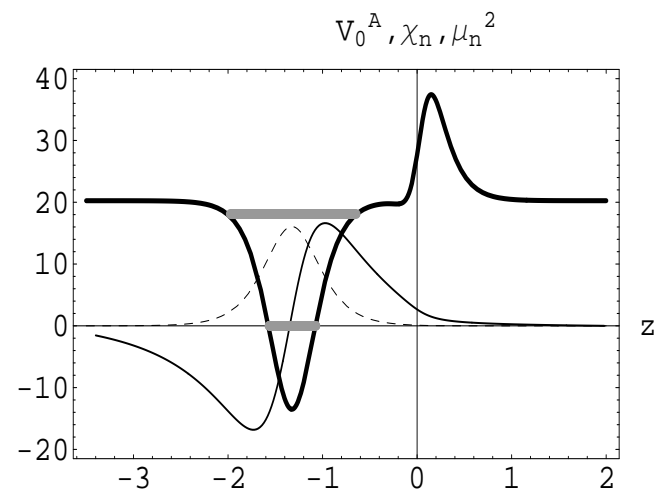

(b) $a=3.818$

Figure 5: The shapes of the potential $V_{0}^{A}(z)$ (thick lines), KK modes $\chi_{n}$ (dashed lines and thin lines) and the mass spectrum $\mu_{n}^{2}$ (thick gray lines) for $d S$ brane with $\beta=3, \delta=1 / 2$, and $a=2$, 3.818 .

\subsection{Spin 1 vector fields}

Next we turn to spin 1 vector fields. We begin with the 5D action of a vector field

$$
S_{1}=-\frac{1}{4} \int d^{5} x \sqrt{-g} g^{M N} g^{R S} F_{M R} F_{N S}
$$

where $F_{M N}=\partial_{M} A_{N}-\partial_{N} A_{M}$ as usual. From this action and the background geometry (2.2), the equations of motion $\frac{1}{\sqrt{-g}} \partial_{M}\left(\sqrt{-g} g^{M N} g^{R S} F_{N S}\right)=0$ are reduced to

$$
\begin{aligned}
\frac{1}{\sqrt{-\hat{g}}} \partial_{\nu}\left(\sqrt{-\hat{g}} \hat{g}^{\nu \rho} \hat{g}^{\mu \lambda} F_{\rho \lambda}\right)+\hat{g}^{\mu \lambda} e^{-A} \partial_{z}\left(e^{A} F_{4 \lambda}\right) & =0, \\
\partial_{\mu}\left(\sqrt{-\hat{g}} \hat{g}^{\mu \nu} F_{\nu 4}\right) & =0 .
\end{aligned}
$$

We assume that $A_{4}$ is $Z_{2}$-odd with respect to the extra dimension $z$, which results in that $A_{4}$ has no zero mode in the effective $4 \mathrm{D}$ theory. Furthermore, in order to consistent with the gauge invariant equation $\oint d z A_{4}=0$, we use gauge freedom to choose $A_{4}=0$. Under the assumption, the action $(3.16)$ is reduced to

$$
S_{1}=-\frac{1}{4} \int d^{5} x \sqrt{-g}\left\{g^{\mu \alpha} g^{\nu \beta} F_{\mu \nu} F_{\alpha \beta}+2 e^{-A} g^{\mu \nu} \partial_{z} A_{\mu} \partial_{z} A_{\nu}\right\} .
$$

Then, with the decomposition of the vector field $A_{\mu}(x, z)=\sum_{n} a_{\mu}^{(n)}(x) \rho_{n}(z) e^{A / 2}$, and importing the orthonormality condition

$$
\int_{-\infty}^{\infty} d z \rho_{m}(z) \rho_{n}(z)=\delta_{m n}
$$

the action (3.19) is read

$$
S_{1}=\sum_{n} \int d^{4} x \sqrt{-\hat{g}}\left(-\frac{1}{4} \hat{g}^{\mu \alpha} \hat{g}^{\nu \beta} f_{\mu \nu}^{(n)} f_{\alpha \beta}^{(n)}-\frac{1}{2} \mu_{n}^{2} \hat{g}^{\mu \nu} a_{\mu}^{(n)} a_{\nu}^{(n)}\right),
$$


where $f_{\mu \nu}^{(n)}=\partial_{\mu} a_{\nu}^{(n)}-\partial_{\nu} a_{\mu}^{(n)}$ is the 4-dimensional field strength tensor, and it has been required that the $\rho_{n}(z)$ satisfies the following Schrödinger equation

$$
\left[-\partial_{z}^{2}+V_{1}(z)\right] \rho_{n}(z)=\mu_{n}^{2} \rho_{n}(z)
$$

where the mass-independent potential is given by

$$
V_{1}^{S}(z)=\frac{\beta^{2}}{4 \delta}\left(\delta-(2+\delta) \operatorname{sech}^{2}(\beta z / \delta)\right)
$$

and

$$
\begin{aligned}
V_{1}^{A}(z)= & \frac{\beta^{2}}{4}+\frac{3 a \beta^{2} \operatorname{sech} 2 \beta z \tanh 2 \beta z}{2(\beta+a \arctan \tanh \beta z)} \\
& -\frac{\beta^{2}\left(5 \beta^{2}-3 a^{2}+5 a(2 \beta+a \arctan \tanh \beta z) \arctan \tanh \beta z\right)}{4(\beta+a \arctan \tanh \beta z)^{2} \cosh ^{2}(2 \beta z)}, \quad\left(\delta=\frac{1}{2}\right)
\end{aligned}
$$

for the symmetric and asymmetric $d S$ brane world solutions given in previous section, respectively. The asymmetric potential at the limit $a \rightarrow 0$ (3.24) is reduced to the symmetric one $(3.23)$ with $\delta=1 / 2$ :

$$
V_{1}^{S}(z)=\frac{\beta^{2}}{4}\left(1-5 \operatorname{sech}^{2}(2 \beta z)\right)
$$

The symmetric potential (3.23) for arbitrary $0<\delta<1$ has a minimum $-\frac{\beta^{2}}{2 \delta}$ at $z=0$ and a maximum $\beta^{2} / 4$ at $z= \pm \infty$. Eq. (3.22) with this potential can be turned into the following Schrödinger equation with a modified Pöschl-Teller potential:

$$
\left[-\partial_{z}^{2}-q(q-1) p^{2} \operatorname{sech}^{2}(p z)\right] \chi_{n}=E_{n} \chi_{n},
$$

where $p=\beta / \delta, q=1+\delta / 2$ and $E_{n}=\mu_{n}^{2}-\frac{1}{4} \delta^{2} p^{2}$. The energy spectrum of bound states is found to be $E_{n}=-p^{2}(q-1-n)$ or

$$
\mu_{n}^{2}=n(\delta-n) \frac{\beta^{2}}{\delta^{2}}, \quad n \in \mathbb{Z}, 0 \leq n<\frac{1}{2} \delta .
$$

For $0<\delta<1$, we get only one bound state, i.e., the normalized zero mode

$$
\rho_{0}(z)=\sqrt{\frac{\beta \Gamma\left(\frac{1}{2}+\frac{\delta}{2}\right)}{\delta \sqrt{\pi} \Gamma\left(\frac{\delta}{2}\right)}} \operatorname{sech}^{\delta / 2}(\beta z / \delta)
$$

with $\mu_{0}=0$. There is a mass gap between the zero mode and the first excited mode. The continuous spectrum starts with $\mu^{2}=\frac{1}{4} \beta^{2}$ and asymptotically turn into plane waves, which represent delocalized KK massive vectors.

For the asymmetric $d S$ brane, the asymmetric potential (3.24) has a negative minimum value at some $z_{0}$ and the asymptotic behavior: $V_{1}^{A}(z= \pm \infty)=\frac{1}{4} \beta^{2}$, which implies that there is also a mass gap. The normalizable massless mode $\rho_{0}(z)$ with $\mu^{2}=0$ is found to be

$$
\rho_{0}(z) \propto\left(\frac{\beta^{2} \operatorname{sech} 2 \beta z}{[\beta+a \arctan (\tanh \beta z)]^{2}}\right)^{1 / 4} .
$$




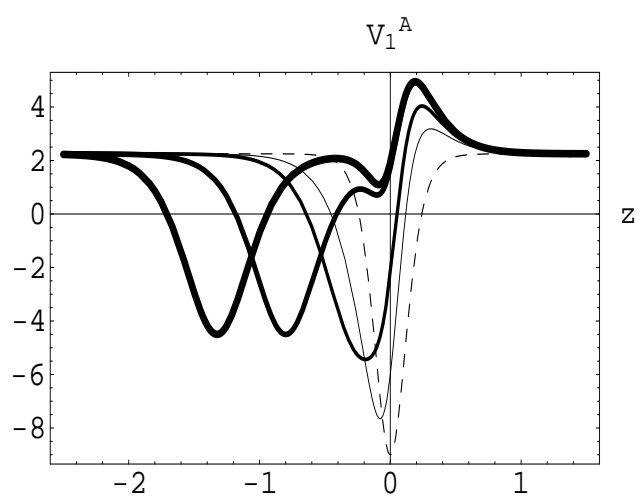

Figure 6: The shapes of the potential $V_{1}^{A}(z)$ for the asymmetric $d S$ brane. The parameters are set to $\beta=3, \delta=1 / 2, a=0$ for dashed line, and $a=2,3,3.78,3.818$ for solid lines with thickness increases with $a$.

This zero mode is the ground state since it has no node. For the limit $a \rightarrow 0$, the massless mode (3.29) is reduced to (3.28) but with $\delta=1 / 2$. Now, we also ask the question: are there other bound states except the zero mode? Since there is no massive bound state for the symmetric potential (3.25), we can conclude that the answer is also no for small asymmetric factor $a$. Just as the case of scalar, the absolute value of the minimum asymmetric potential decreases with the increase of the asymmetry (see Fig. 6). However, we do not find massive bound states by numerical method even for large $a$.

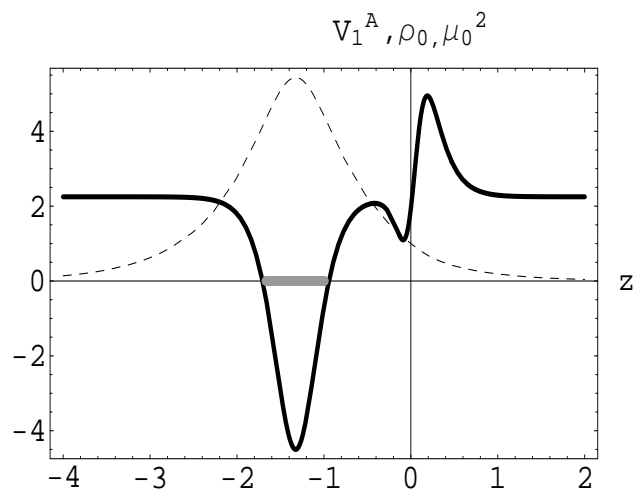

Figure 7: The shapes of the potential $V_{1}^{A}(z)$ (thick line), zero mode (dashed line) and the mass spectrum (thick gray line) for $d S$ brane with $\beta=3, \delta=1 / 2$ and $a=3.818$.

It was shown in the RS model in $A d S_{5}$ space that a spin 1 vector field is not localized neither on a brane with positive tension nor on a brane with negative tension so the DvaliShifman mechanism [39] must be considered for the vector field localization [20]. Here, it is turned out that a vector field can be localized on the $d S$ thick branes and we do not need to introduce additional mechanism for the vector field localization in the case at hand. For $0<\delta<1$, we get only one bound state which is the massless mode. Furthermore, there exists a mass gap between the bound ground state and the first exited state. 


\subsection{Spin $1 / 2$ fermion fields}

In five dimensions, fermions are four component spinors and their Dirac structure is described by $\Gamma^{M}=e_{\bar{M}}^{M} \Gamma^{\bar{M}}$ with $e_{\bar{M}}^{M}$ being the vielbein and $\left\{\Gamma^{M}, \Gamma^{N}\right\}=2 g^{M N}$. In this paper, $\bar{M}, \bar{N}, \cdots=0,1,2,3,5$ and $\bar{\mu}, \bar{\nu}, \cdots=0,1,2,3$ denote the $5 \mathrm{D}$ and 4D local Lorentz indices respectively, and $\Gamma^{\bar{M}}$ are the flat gamma matrices in five dimensions. In our set-up, the vielbein is given by

$$
e_{M}^{\bar{M}}=\left(\begin{array}{cc}
\mathrm{e}^{A} \hat{e}_{\mu}^{\bar{\nu}} & 0 \\
0 & \mathrm{e}^{A}
\end{array}\right)
$$

$\Gamma^{M}=\mathrm{e}^{-A}\left(\hat{e}_{\bar{\nu}}^{\mu} \gamma^{\bar{\nu}}, \gamma^{5}\right)=\mathrm{e}^{-A}\left(\gamma^{\mu}, \gamma^{5}\right)$, where $\gamma^{\mu}=\hat{e}_{\bar{\nu}}^{\mu} \gamma^{\bar{\nu}}, \gamma^{\bar{\nu}}$ and $\gamma^{5}$ are the usual flat gamma matrices in the $4 \mathrm{D}$ Dirac representation. The Dirac action of a massless spin $1 / 2$ fermion coupled to the scalar is

$$
S_{1 / 2}=\int d^{5} x \sqrt{-g}\left(\bar{\Psi} \Gamma^{M}\left(\partial_{M}+\omega_{M}\right) \Psi-\eta \bar{\Psi} F(\phi) \Psi\right),
$$

where the spin connection is defined as $\omega_{M}=\frac{1}{4} \omega_{M}^{\bar{M}} \bar{N} \Gamma_{\bar{M}} \Gamma_{\bar{N}}$ and

$$
\begin{aligned}
\omega_{M}^{\bar{M} \bar{N}} & =\frac{1}{2} e^{N \bar{M}}\left(\partial_{M} e_{N}^{\bar{N}}-\partial_{N} e_{M}^{\bar{N}}\right) \\
& -\frac{1}{2} e^{N \bar{N}}\left(\partial_{M} e_{N}^{\bar{M}}-\partial_{N} e_{M}^{\bar{M}}\right) \\
& -\frac{1}{2} e^{P \bar{M}} e^{Q \bar{N}}\left(\partial_{P} e_{Q \bar{R}}-\partial_{Q} e_{P \bar{R}}\right) e_{M}^{\bar{R}} .
\end{aligned}
$$

The non-vanishing components of the spin connection $\omega_{M}$ for the background metric (2.2) are

$$
\omega_{\mu}=\frac{1}{2}\left(\partial_{z} A\right) \gamma_{\mu} \gamma_{5}+\hat{\omega}_{\mu}
$$

where $\mu=0,1,2,3$ and $\hat{\omega}_{\mu}=\frac{1}{4} \bar{\omega}_{\mu}^{\bar{\mu} \bar{\nu}} \Gamma_{\bar{\mu}} \Gamma_{\bar{\nu}}$ is the spin connection derived from the metric $\hat{g}_{\mu \nu}(x)=\hat{e}_{\mu}{ }^{\bar{\mu}} \hat{e}_{\nu} \eta_{\bar{\mu}}$. Then the equation of motion is given by

$$
\left\{\gamma^{\mu}\left(\partial_{\mu}+\hat{\omega}_{\mu}\right)+\gamma^{5}\left(\partial_{z}+2 \partial_{z} A\right)-\eta \mathrm{e}^{A} F(\phi)\right\} \Psi=0,
$$

where $\gamma^{\mu}\left(\partial_{\mu}+\hat{\omega}_{\mu}\right)$ is the Dirac operator on the brane.

Now we study the above 5-dimensional Dirac equation, and write the spinor in terms of 4-dimensional effective fields. Because of the Dirac structure of the fifth gamma matrix $\gamma^{5}$, we expect that the left- and right-handed projections of the four dimensional part to behave differently. From the equation of motion (3.34), we will search for the solutions of the general chiral decomposition

$$
\Psi(x, z)=\mathrm{e}^{-2 A}\left(\sum_{n} \psi_{L n}(x) \alpha_{L n}(z)+\sum_{n} \psi_{R n}(x) \alpha_{R n}(z)\right)
$$

with $\psi_{L n}(x)=-\gamma^{5} \psi_{L n}(x)$ and $\psi_{R n}(x)=\gamma^{5} \psi_{R n}(x)$ the left-handed and right-handed components of a $4 \mathrm{D}$ Dirac field. Here, to obtain the equations for the basis functions $\psi_{L n}(x)$ 
and $\psi_{R n}(x)$, we assume that $\psi_{L}(x)$ and $\psi_{R}(x)$ satisfy the $4 \mathrm{D}$ massive Dirac equations $\gamma^{\mu}\left(\partial_{\mu}+\hat{\omega}_{\mu}\right) \psi_{L n}(x)=\mu_{n} \psi_{R_{n}}(x)$ and $\gamma^{\mu}\left(\partial_{\mu}+\hat{\omega}_{\mu}\right) \psi_{R n}(x)=\mu_{n} \psi_{L_{n}}(x)$. Then the KK modes $\alpha_{L n}(z)$ and $\alpha_{R n}(z)$ satisfy the following coupled equations

$$
\begin{aligned}
& {\left[\partial_{z}+\eta \mathrm{e}^{A} F(\phi)\right] \alpha_{L n}(z)=\mu_{n} \alpha_{R n}(z),} \\
& {\left[\partial_{z}-\eta \mathrm{e}^{A} F(\phi)\right] \alpha_{R n}(z)=-\mu_{n} \alpha_{L n}(z),}
\end{aligned}
$$

i.e.,

$$
\begin{aligned}
& {\left[\partial_{z}-\eta \mathrm{e}^{A} F(\phi)\right]\left[\partial_{z}+\eta \mathrm{e}^{A} F(\phi)\right] \alpha_{L n}(z)=-\mu_{n}^{2} \alpha_{L n}(z),} \\
& {\left[\partial_{z}+\eta \mathrm{e}^{A} F(\phi)\right]\left[\partial_{z}-\eta \mathrm{e}^{A} F(\phi)\right] \alpha_{R n}(z)=-\mu_{n}^{2} \alpha_{R n}(z)}
\end{aligned}
$$

Hence, we get the Schrödinger-like equations for the left and right chiral fermions

$$
\begin{aligned}
& \left(-\partial_{z}^{2}+V_{L}(z)\right) \alpha_{L n}=\mu_{n}^{2} \alpha_{L n} \\
& \left(-\partial_{z}^{2}+V_{R}(z)\right) \alpha_{R n}=\mu_{n}^{2} \alpha_{R n}
\end{aligned}
$$

where the mass-independent potentials are given by

$$
\begin{aligned}
V_{L}(z) & =\mathrm{e}^{2 A} \eta^{2} F^{2}(\phi)-\mathrm{e}^{A} \eta \partial_{z} F(\phi)-\left(\partial_{z} A\right) \mathrm{e}^{A} \eta F(\phi), \\
V_{R}(z) & =\left.V_{L}(z)\right|_{\eta \rightarrow-\eta} .
\end{aligned}
$$

In order to obtain the standard $4 \mathrm{D}$ action for the massive chiral fermions:

$$
\begin{aligned}
S_{1 / 2} & =\int d^{5} x \sqrt{-g} \bar{\Psi}\left(\Gamma^{M}\left(\partial_{M}+\omega_{M}\right)-\eta F(\phi)\right) \Psi \\
& =\sum_{n} \int d^{4} x \sqrt{-\hat{g}}\left\{\bar{\psi}_{R n} \gamma^{\mu}\left(\partial_{\mu}+\hat{\omega}_{\mu}\right) \psi_{R n}-\bar{\psi}_{R n} \mu_{n} \psi_{L n}\right\} \\
& +\sum_{n} \int d^{4} x \sqrt{-\hat{g}}\left\{\bar{\psi}_{L n} \gamma^{\mu}\left(\partial_{\mu}+\hat{\omega}_{\mu}\right) \psi_{L n}-\bar{\psi}_{L n} \mu_{n} \psi_{R n}\right\} \\
& =\sum_{n} \int d^{4} x \sqrt{-\hat{g}} \bar{\psi}_{n}\left[\gamma^{\mu}\left(\partial_{\mu}+\hat{\omega}_{\mu}\right)-\mu_{n}\right] \psi_{n},
\end{aligned}
$$

we need the following orthonormality conditions for $\alpha_{L_{n}}$ and $\alpha_{R_{n}}$ :

$$
\begin{aligned}
& \int_{-\infty}^{\infty} \alpha_{L m} \alpha_{L n} d z=\delta_{m n} \\
& \int_{-\infty}^{\infty} \alpha_{R m} \alpha_{R n} d z=\delta_{m n} \\
& \int_{-\infty}^{\infty} \alpha_{L m} \alpha_{R n} d z=0
\end{aligned}
$$

It can be seen that, for the left (right) chiral fermion localization, there must be some kind of scalar-fermion coupling. This situation can be compared with the one in the RS framework [20], where additional localization method [40] was introduced for spin $1 / 2$ 
fields. Furthermore, $F(\phi(z))$ must be an odd function of $\phi(z)$ when we demand that $V_{L}(z)$ or $V_{R}(z)$ is $Z_{2}$-even with respect to the extra dimension $z$. In this paper, we will consider two cases $F(\phi)=\phi$ and $F(\phi)=\sin \left(\frac{\phi}{\phi_{0}}\right) \cos ^{-\delta}\left(\frac{\phi}{\phi_{0}}\right)$ as examples. For $F(\phi)=\phi$, we get a continuous spectrum of KK modes with $\mu^{2} \geq 0$. However, it is shown that even the massless left and right chiral modes can not be localized on the brane. For $F(\phi)=\sin \left(\frac{\phi}{\phi_{0}}\right) \cos ^{-\delta}\left(\frac{\phi}{\phi_{0}}\right)$, there exists a mass gap, and we get some discrete bound modes and a continuous spectrum of KK modes.

\subsubsection{Case I}

For the first case $F(\phi)=\phi$, the explicit forms of the potentials (3.40) are

$$
\begin{aligned}
V_{L}^{S}(z)= & \eta^{2} \phi_{0}^{2} \cosh ^{-2 \delta}\left(\frac{\beta z}{\delta}\right) \arctan ^{2} \sinh \left(\frac{\beta z}{\delta}\right) \\
& +\frac{\eta \beta \phi_{0}}{\delta} \cosh ^{-1-\delta}\left(\frac{\beta z}{\delta}\right)\left[\delta \sinh \left(\frac{\beta z}{\delta}\right) \arctan \sinh \left(\frac{\beta z}{\delta}\right)-1\right], \\
V_{R}^{S}(z)= & \left.V_{L}^{S}(z)\right|_{\eta \rightarrow-\eta},
\end{aligned}
$$

and

$$
\begin{aligned}
V_{L}^{A}(z)= & \frac{\eta \beta^{2} \phi_{0} \cosh ^{-3 / 2}(2 \beta z)}{\left(\beta+a \arctan ^{2} \tanh (\beta z)\right)^{2}}[a \arctan \sinh (2 \beta z) \\
& +\eta \phi_{0} \sqrt{\cosh (2 \beta z)} \arctan ^{2} \sinh (2 \beta z) \quad\left(\delta=\frac{1}{2}\right) \\
& +(\beta+a \arctan \tanh (\beta z))(\sinh (2 \beta z) \arctan \sinh (2 \beta z)-2)], \\
V_{R}^{A}(z)= & \left.V_{L}^{A}(z)\right|_{\eta \rightarrow-\eta},
\end{aligned}
$$

for the symmetric and asymmetric $d S$ brane world solutions, respectively.
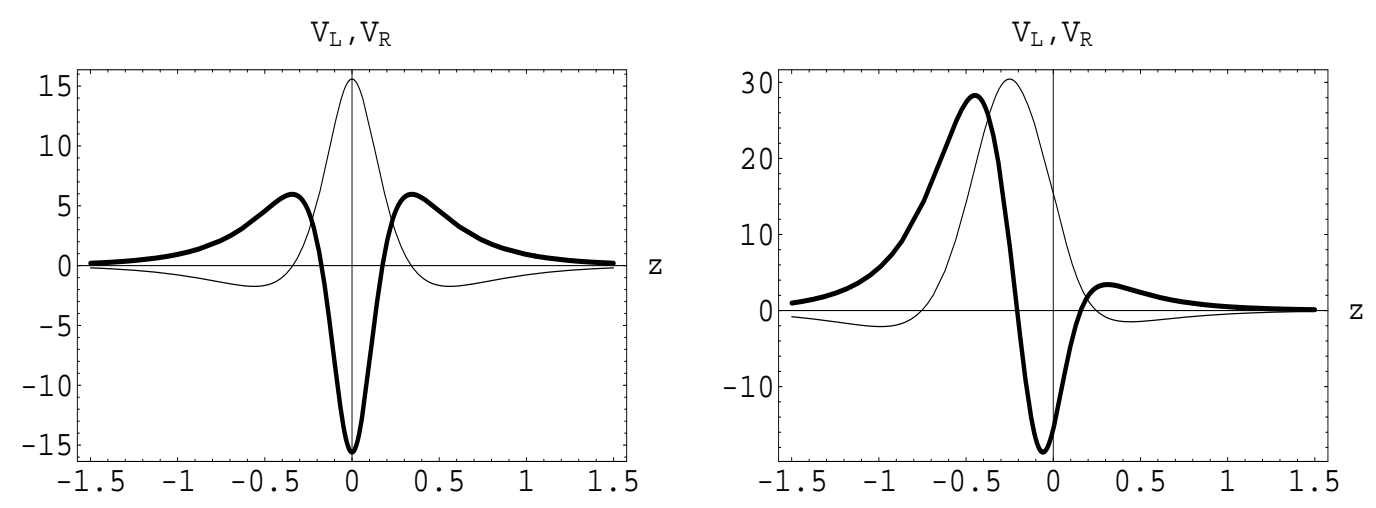

Figure 8: The shapes of the potentials $V_{L}$ (thick lines), $V_{R}$ (thin lines) for left and right chiral fermions for the case $F(\phi)=\phi$. The parameters are set to $\delta=1 / 2, \eta=3, \beta=3, a=0$ (left) and $a=3$ (right).

All potentials have the asymptotic behavior: $V_{L, R}(z \rightarrow \pm \infty) \rightarrow 0$. But for a given coupling constant $\eta$ and the parameter $\beta$, the values of the potentials for the left and right 
chiral fermions at $z=0$ are opposite. The shapes of the potentials are shown in Fig. 8 for given values of positive $\eta$ and $\beta$. It can be seen that $V_{L}(z)$ is indeed a modified volcano type potential. Hence, the potential provides no mass gap to separate the fermion zero mode from the excited KK modes, and there exists a continuous gapless spectrum of the KK modes for both the left chiral and right chiral fermions.

For positive $\beta$ and $\eta$, only the potential for left chiral fermions has a negative value at the location of the brane, which could trap the left chiral fermion zero mode solved from (3.36a) by setting $\mu_{0}=0$ :

$$
\alpha_{L 0}(z) \propto \exp \left(-\eta \int^{z} d z^{\prime} \mathrm{e}^{A\left(z^{\prime}\right)} \phi\left(z^{\prime}\right)\right) .
$$

In order to check the normalization condition (3.42) for the zero mode (3.49), we need to check whether the inequality

$$
\int d z \exp \left(-2 \eta \int^{z} d z^{\prime} \mathrm{e}^{A\left(z^{\prime}\right)} \phi\left(z^{\prime}\right)\right)<\infty
$$

is satisfied. For the integral $\int d z \mathrm{e}^{A} \phi$, we only need to consider the asymptotic characteristic of the function $\mathrm{e}^{A} \phi$ for $z \rightarrow \infty$. For symmetric $d S$ brane case, noting that $\arctan (\sinh z) \rightarrow$ $\pi / 2$ when $z \rightarrow \infty$, we have

$$
\begin{aligned}
& \mathrm{e}^{A} \phi=\phi_{0} \cosh ^{-\delta}\left(\frac{\beta z}{\delta}\right) \arctan \left(\sinh \frac{\beta z}{\delta}\right) \rightarrow \frac{\pi}{2} \phi_{0} 2^{\delta} \mathrm{e}^{-\beta z}, \\
& \exp \left(-2 \eta \int d z \mathrm{e}^{A} \phi\right) \rightarrow \exp \left(2^{\delta} \pi \eta \phi_{0} \mathrm{e}^{-\beta z} / \beta\right) \rightarrow 1,
\end{aligned}
$$

which indicates that the normalization condition $(3.50)$ is not satisfied and the zero mode of the left chiral fermions can not be localized on the brane. For asymmetric case, we can also get the same conclusion. This is different from the conclusion obtained in Refs. [30, 41], where the zero mode of the left chiral fermions can be localized on the Branes in the Background of Sine-Gordon Kinks.

\subsubsection{Case II}

For the case $F(\phi)=\sin \left(\frac{\phi}{\phi_{0}}\right) \cos ^{-\delta}\left(\frac{\phi}{\phi_{0}}\right)$, the potentials $(3.40)$ are

$$
\begin{aligned}
& V_{L}^{S}(z)=\eta\left(\eta-\frac{\beta+\delta \eta}{\delta} \operatorname{sech}^{2}(\beta z / \delta)\right), \\
& V_{R}^{S}(z)=\left.V_{L}^{S}(z)\right|_{\eta \rightarrow-\eta},
\end{aligned}
$$

and

$$
\begin{aligned}
V_{L}^{A}(z) & =\frac{\eta \beta^{2} \operatorname{sech}(2 \beta z) \tanh (2 \beta z)(a+\eta \sinh (2 \beta z))}{(\beta+a \arctan \tanh (\beta z))^{2}}-\frac{2 \eta \beta^{2} \operatorname{sech}^{2}(2 \beta z)}{\beta+a \arctan \tanh (\beta z)}, \\
V_{R}^{A}(z) & =\left.V_{L}^{A}(z)\right|_{\eta \rightarrow-\eta},
\end{aligned}
$$

for the symmetric and asymmetric $d S$ brane world solutions, respectively. 


\section{Symmetric $d S$ brane}

We first investigate the case of symmetric $d S$ brane. The values of the corresponding potentials (3.53) and (3.54) at $y=0$ and $y= \pm \infty$ are given by

$$
\begin{gathered}
V_{L}^{S}(0)=-V_{R}^{S}(0)=-\frac{\beta \eta}{\delta}, \\
V_{L}^{S}( \pm \infty)=V_{R}^{S}( \pm \infty)=\eta^{2},
\end{gathered}
$$

i.e., both potentials have same asymptotic behavior when $y \rightarrow \pm \infty$, but opposite behavior at the origin $z=0$. The shapes of the two potentials are shown in Figs. 9 and 10 for different values of $\beta$ and $\eta$, respectively.
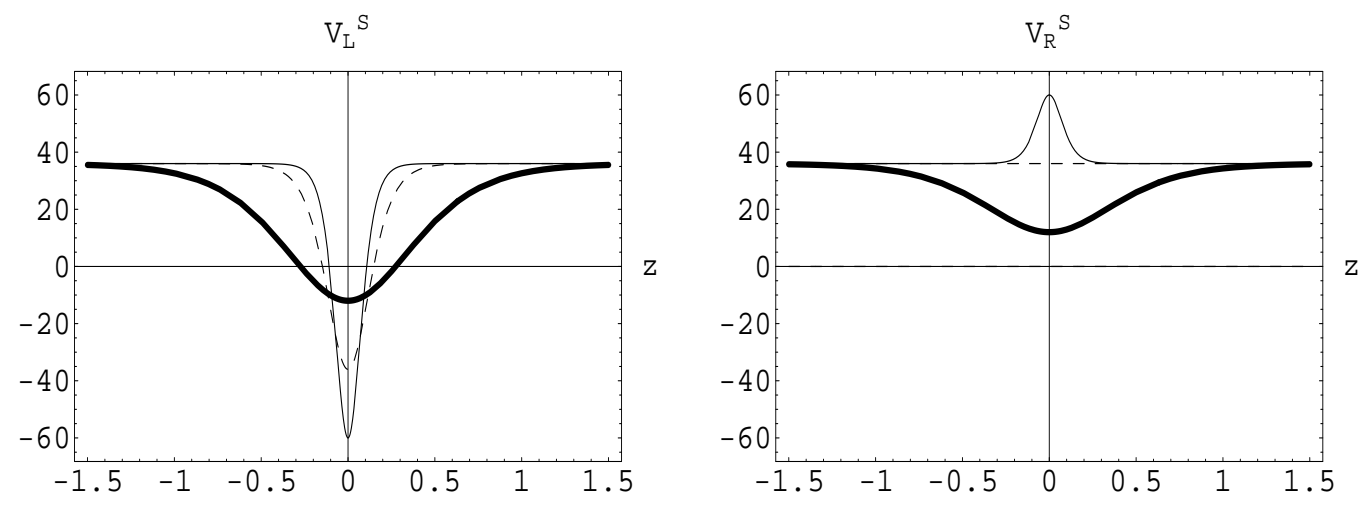

Figure 9: The shapes of the potentials $V_{L}^{S}$ (left) and $V_{R}^{S}$ (right) of left and right chiral fermions for the symmetric $d S$ brane with different $\beta$. The parameters are set to $\delta=1 / 2, \eta=6$, and $\beta=1$ for thick lines, $\beta=3$ for dashed lines and $\beta=5$ for thin lines.
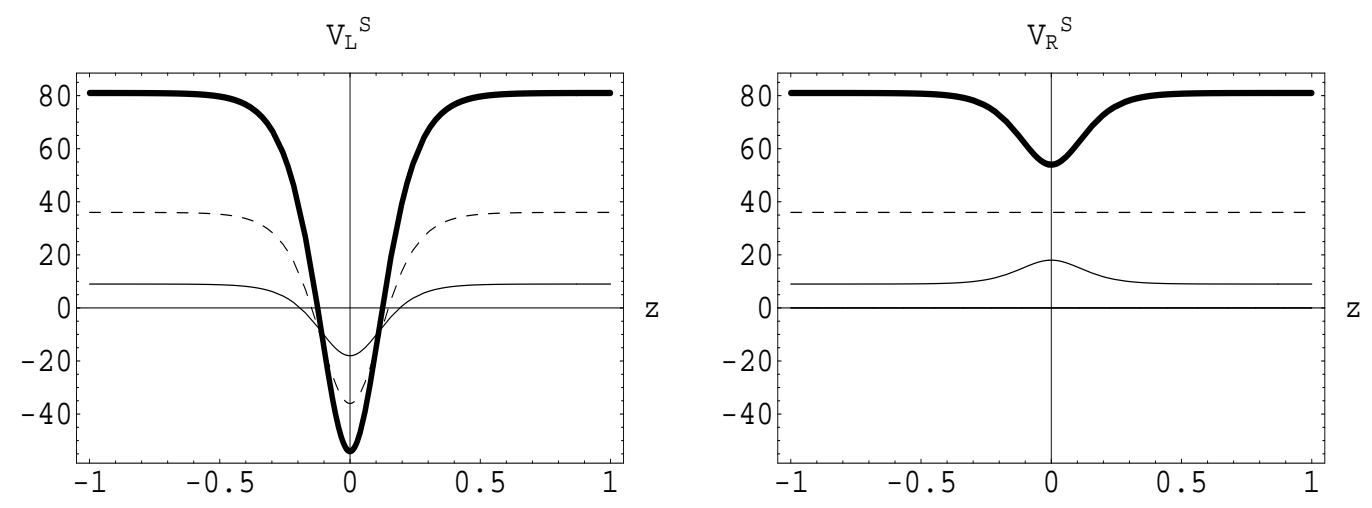

Figure 10: The shapes of the potentials $V_{L}^{S}$ (left) and $V_{R}^{S}$ (right) of left and right chiral fermions for the symmetric $d S$ brane with different $\eta$. The parameters are set to $\delta=1 / 2, \beta=3$, and $\eta=9$ for thick lines, $\eta=6$ for dashed lines and $\eta=3$ for thin lines.

Note that, for a positive coupling constant $\eta$, the potential for left chiral fermions has a negative value at the location of the brane and a positive value far away from the brane 
along the extra dimension, which can always trap the left chiral fermion zero mode:

$$
\alpha_{L 0}^{S}=\left[\frac{\beta \Gamma\left(\frac{\beta+\delta \eta}{2 \beta}\right)}{\delta \sqrt{\pi} \Gamma\left(\frac{\delta \eta}{2 \beta}\right)}\right]^{\frac{1}{2}} \cosh ^{-\delta \eta / \beta}(\beta z / \delta) . \quad(\eta>0)
$$

The zero mode represents the lowest energy eigenfunction of the Schrödinger equation (3.38) since it has no nodes. The right chiral fermion zero mode for $\eta>0$ is not localized on the brane, which can be seen from the potential $V_{R}^{S}$ in Figs. (9) and (10).

It is clear that for $\mu_{L n}^{2}>\eta^{2}$, we obtain the asymptotic plane waves. The general bound states for the potential $V_{L}^{S}($ (3.53) for left chiral fermions are found to be

$$
\alpha_{L n}^{S} \propto \cosh ^{1+\frac{\delta \eta}{\beta}}\left(\frac{\beta z}{\delta}\right){ }_{2} F_{1}\left(a_{n}, b_{n} ; \frac{1}{2} ;-\sinh ^{2}(\beta z / \delta)\right),
$$

for even $n$ and

$$
\alpha_{L n}^{S} \propto \cosh ^{1+\frac{\delta \eta}{\beta}}\left(\frac{\beta z}{\delta}\right) \sinh \left(\frac{\beta z}{\delta}\right){ }_{2} F_{1}\left(a_{n}+\frac{1}{2}, b_{n}+\frac{1}{2} ; \frac{3}{2} ;-\sinh ^{2}(\beta z / \delta)\right),
$$

for odd $n$, where ${ }_{2} F_{1}$ is the hypergeometric function, the parameters $a_{n}$ and $b_{n}$ are given by

$$
a_{n}=\frac{1}{2}(n+1), \quad b_{n}=\frac{\delta \eta}{\beta}-\frac{1}{2}(n-1) .
$$

The corresponding mass spectrum of the bound states is

$$
\mu_{L n}^{2}=\frac{\beta(2 \delta \eta-\beta n) n}{\delta^{2}}, \quad\left(\eta>0, n=0,1,2, \ldots<\frac{\delta \eta}{\beta}\right) .
$$

It shows that the ground state always belongs to the spectrum of $V_{L}^{S}(z)$ for positive $\eta$, which is just the zero mode (3.59) with $\mu_{L 0}=0$. Since the ground state has the lowest mass square $\mu_{L 0}^{2}=0$, there is no tachyonic left chiral fermion modes. Here, we suppose the number of bound states for left chiral fermions is $N_{L}$. If $0<\eta \leq \beta / \delta$, there is only one bound state $\left(N_{L}=1\right)$, i.e., the zero mode (3.59). In order to get bound exited states $\left(N_{L} \geq 2\right)$, we need the condition $\eta>\beta / \delta$.

In the case $\eta>0$, the potential $V_{R}^{S}(z)=\eta\left(\eta+\frac{\beta-\delta \eta}{\delta} \operatorname{sech}^{2}(\beta z / \delta)\right)$ for right chiral fermions is always positive near the location of the brane, which shows that it can not trap the right chiral zero mode. For the case $0<\eta<\beta / \delta$, we have $V_{R}^{S}(0) \geq V_{R}^{S}( \pm \infty)>0$, which shows that there is no any bound state for the potential of right chiral fermions. For the special value $\eta=\beta / \delta$, the potential $V_{R}^{S}$ is a positive constant: $V_{R}^{S}(z)=\eta^{2}=\beta^{2} / \delta^{2}$, and there is still no any bound state. However, provided $\eta>\beta / \delta$, we will get a potential well since $V_{R}^{S}(0)<V_{R}^{S}( \pm \infty)$ (see Figs. 9 and 10), which indicates that there may be some bound states, but none of them is zero mode. The general bound states for the potential $V_{R}^{S}$ are

$$
\alpha_{R n}^{S} \propto \cosh ^{\frac{\delta \eta}{\beta}}\left(\frac{\beta z}{\delta}\right){ }_{2} F_{1}\left(\frac{1+n}{2}, \frac{\delta \eta}{\beta}-\frac{1+n}{2} ; \frac{1}{2} ;-\sinh ^{2}(\beta z / \delta)\right)
$$


for even $n$ and

$$
\alpha_{R n}^{S} \propto \cosh ^{\frac{\delta \eta}{\beta}}\left(\frac{\beta z}{\delta}\right) \sinh \left(\frac{\beta z}{\delta}\right){ }_{2} F_{1}\left(1+\frac{n}{2}, \frac{\delta \eta}{\beta}-\frac{n}{2} ; \frac{3}{2} ;-\sinh ^{2}(\beta z / \delta)\right)
$$

for odd $n$. The corresponding mass spectrum is

$$
\mu_{R n}^{2}=\frac{(n+1) \beta(2 \delta \eta-(n+1) \beta)}{\delta^{2}}, \quad\left(\eta>\frac{\beta}{\delta}, n=0,1,2, \ldots<\frac{\delta \eta}{\beta}-1\right) .
$$

By comparing with the mass spectrum of left chiral fermions (3.66), we come to the conclusion that the number of bound states of right chiral fermions $N_{R}$ is one less than that of left ones, i.e., $N_{R}=N_{L}-1$. If $0<\eta \leq \beta / \delta$, there is only one left chiral fermion bound state (the zero mode). If $\eta>\beta / \delta$, there are $N_{L}\left(N_{L} \geq 2\right)$ left chiral fermion bound states and $N_{L}-1$ right chiral fermion bound states. The ground state for right chiral fermions is

$$
\alpha_{R 0}^{S}=\left[\frac{\beta \Gamma\left(\frac{\delta \eta}{2 \beta}\right)}{\delta \sqrt{\pi} \Gamma\left(\frac{\delta \eta}{2 \beta}-\frac{1}{2}\right)}\right]^{\frac{1}{2}} \cosh ^{1-\frac{\delta \eta}{\beta}}\left(\frac{\beta z}{\delta}\right), \quad\left(\eta>\frac{\beta}{\delta}\right)
$$

which is not zero mode any more because the mass is determined by $\mu_{R 0}^{2}=\beta(2 \delta \eta-\beta) / \delta^{2}>$ $\beta^{2} / \delta^{2}>0$. In Figs. 13(a) and 13(b) we plot the potentials, the mass spectra and some bound states of left and right chiral fermions. For the case $\delta=1 / 2, \beta=1, \eta=11$, there are 6 and 5 bound states for the left and the right chiral fermions respectively and the mass spectra are

$$
\begin{aligned}
& \mu_{L n}^{2}=\{0,40,72,96,112,120\} \cup[121, \infty), \\
& \mu_{R n}^{2}=\{40,72,96,112,120\} \cup[121, \infty) .
\end{aligned}
$$

\section{Asymmetric $d S$ brane}

Now we turn to the case of asymmetric $d S$ brane, for which the corresponding potentials (3.55) and (3.56) are obviously asymmetric and the solution of the bound states and mass spectrum is very complex. The values of the potentials at $z=0, \pm \infty$ are given by

$$
\begin{gathered}
V_{L}^{A}(0)=-V_{R}^{A}(0)=-2 \beta \eta, \\
V_{L}^{A}(+\infty)=V_{R}^{A}(+\infty)=\frac{16 \beta^{2} \eta^{2}}{(a \pi+4 \beta)^{2}}<\eta^{2}, \\
V_{L}^{A}(-\infty)=V_{R}^{A}(-\infty)=\frac{16 \beta^{2} \eta^{2}}{(a \pi-4 \beta)^{2}}>\eta^{2} .
\end{gathered}
$$

Both potentials have also same asymptotic behavior when $z \rightarrow \pm \infty$. However, compared with the symmetric potentials (3.53) and (3.54), $V_{L, R}^{A}(-\infty)$ increase and $V_{L, R}^{A}(+\infty)$ decrease for positive asymmetric factor $a$, which may reduce the number of the bound states. The shapes of the two potentials are shown in Figs. 11 and 12 for different values of $\beta$ and $\eta$, respectively. Different from the symmetric potentials $V_{L, R}^{S}(z)$, the asymmetric ones $V_{L, R}^{A}(z)$ at $z= \pm \infty$ dependent on the parameter $\beta$ unless the asymmetric factor $a=0$. Hence, even at same $\eta$ and $a$, the limits of $V_{L, R}^{A}(z)$ at $z \rightarrow+\infty$ and $z \rightarrow-\infty$ are different 
for different $\beta$ (see Fig. 11). For positive $\eta$, the right chiral fermion zero mode does not exist, but the left one is always exist and can be solved as

$$
\alpha_{L 0}^{A}(z) \propto \exp \left(-\eta \beta \int^{z} d z^{\prime} \frac{\tanh \left(2 \beta z^{\prime}\right)}{\beta+a \arctan \tanh \left(\beta z^{\prime}\right)}\right) .
$$
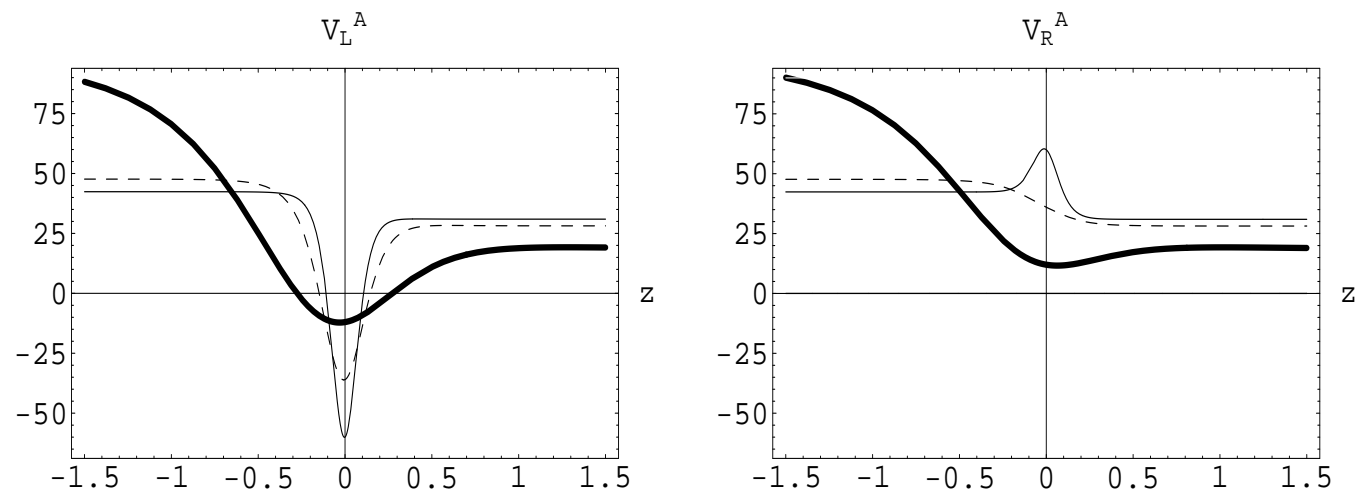

Figure 11: The shapes of the potentials $V_{L}^{A}$ (left) and $V_{R}^{A}$ (right) of left and right chiral fermions for the asymmetric $d S$ brane with different $\beta$. The parameters are set to $\delta=1 / 2, \eta=6, a=0.5$, and $\beta=1$ for thick lines, $\beta=3$ for dashed lines and $\beta=5$ for thin lines.
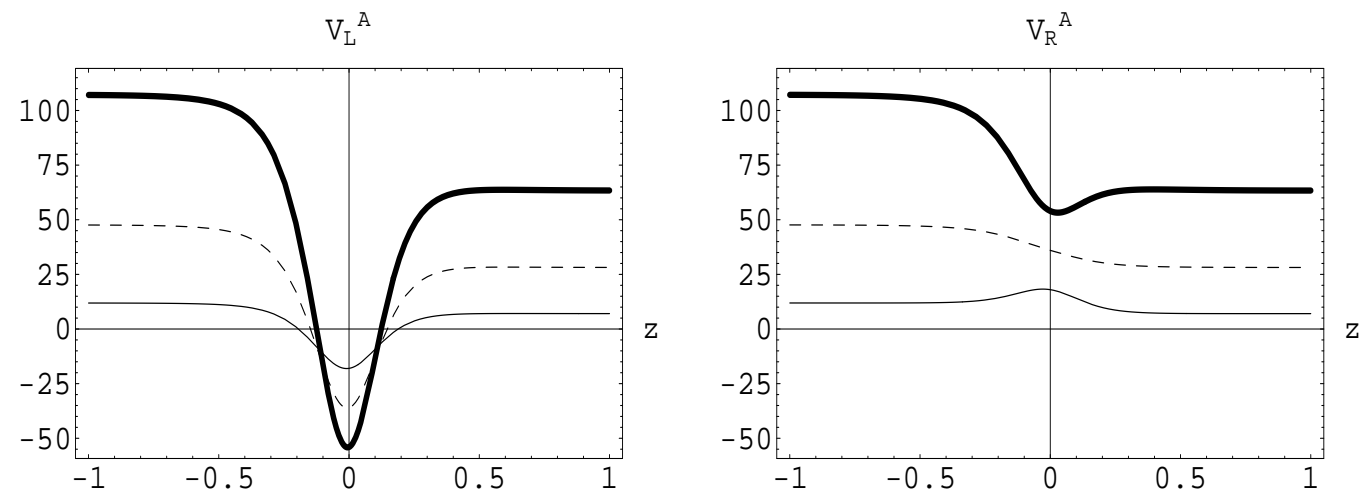

Figure 12: The shapes of the potentials $V_{L}^{A}$ (left) and $V_{R}^{A}$ (right) of left and right chiral fermions for the asymmetric $d S$ brane with different $\eta$. The parameters are set to $\delta=1 / 2, \beta=3, a=0.5$, and $\eta=9$ for thick lines, $\eta=6$ for dashed lines and $\eta=3$ for thin lines.

For $\mu_{n}^{2}>16 \beta^{2} \eta^{2} /(a \pi+4 \beta)^{2}$, we obtain the continuum of asymptotic plane waves. In order to obtain acceptable normalizable modes, $\mu_{n}^{2}$ should be limited in the interval $\left[0,16 \beta^{2} \eta^{2} /(a \pi+4 \beta)^{2}\right)$. Although the analytic massive modes can not be solved because of the complexity of the potentials, we can get the numerical solutions. The mass spectra are listed in Tab. 1 for left chiral fermions and Tab. 2 for right ones for some given parameters. We also plot the potentials, mass spectra and part of the eigenfunctions in Fig. 13. From these tables and Eq. (3.70), we can draw a conclusion: the number of the bound states increases with the coupling constant $\eta$ but decreases with the asymmetric factor $a$. 


\begin{tabular}{|c|c|c|r|l|}
\hline$a$ & $N_{L}$ & $V_{L}^{A}(+\infty)$ & $V_{L}^{A}(-\infty)$ & Mass spectrum $\mu_{L n}^{2}$ of bound states \\
\hline \hline 0.00 & 6 & 121.0 & 121.0 & $\{0,40.00,72.00,96.00,112.00,120.00\}$ \\
0.02 & 5 & 117.3 & 124.9 & $\{0,40.00,71.99,95.94,111.74\}$ \\
0.10 & 4 & 104.0 & 142.5 & $\{0,39.95,71.67,94.57\}$ \\
0.25 & 3 & 84.5 & 187.4 & $\{0,39.70,69.95\}$ \\
0.50 & 2 & 62.3 & 328.1 & $\{0,38.79\}$ \\
1.25 & 1 & 30.8 & 363203.8 & $\{0\}$ \\
\hline
\end{tabular}

Table 1: Mass spectrum of bound states for asymmetric potentials $V_{L}^{A}(z)$. The parameters are set to $\delta=1 / 2, \beta=1, \eta=11$. $N_{L}$ presents the number of bound states for left chiral fermions.

\begin{tabular}{|c|c|c|r|l|}
\hline$a$ & $N_{R}$ & $V_{R}^{A}(+\infty)$ & $V_{R}^{A}(-\infty)$ & Mass spectrum $\mu_{R n}^{2}$ of bound states \\
\hline \hline 0.00 & 5 & 121.0 & 121.0 & $\{40.00,72.00,96.00,112.00,120.00\}$ \\
0.02 & 4 & 117.3 & 124.9 & $\{40.00,71.99,95.94,111.74\}$ \\
0.10 & 3 & 104.0 & 142.5 & $\{39.95,71.67,94.57\}$ \\
0.25 & 2 & 84.5 & 187.4 & $\{39.70,69.95\}$ \\
0.50 & 1 & 62.3 & 328.1 & $\{38.79\}$ \\
1.25 & 0 & 30.8 & 363203.8 & \{\} \\
\hline
\end{tabular}

Table 2: Mass spectrum of bound states for asymmetric potentials $V_{R}^{A}(z)$. The parameters are set to $\delta=1 / 2, \beta=1, \eta=11 . N_{R}$ presents the number of bound states for right chiral fermions.

To close this section, we make some comments on the issue of the localization of fermions. Localizing the fermions on branes or defects requires us to introduce other interactions besides gravity. More recently, Volkas et al had extensively analyzed localization mechanisms on a domain wall. In particular, in Ref. [24], they proposed a well-defined model to localize the SM, or something close to it, on a domain wall brane. There are some other backgrounds, for example, gauge field [43], supergravity [44, 45] and vortex background [46, 47, 48, 49], could be considered. The topological vortex coupled to fermions may result in chiral fermion zero modes [50].

\section{Conclusion and discussion}

In this paper, by presenting the shapes of the mass-independent potentials of KK modes in the corresponding Schrödinger equations, we have investigated the localization and mass spectra of various matter fields with spin 0,1 and $1 / 2$ on symmetric and asymmetric $d S$ thick branes, where the asymmetric $d S$ thick brane is constructed from the symmetric one by using a same scalar (kink) with different potentials.

For spin 0 scalars and spin 1 vectors, the potentials of KK modes in the corresponding Schrödinger equations are the modified Pöschl-Teller potentials. They have a finite negative well at the location of the brane and a finite positive barrier at each side which doesn't vanishes. Such potentials suggest that there exist a mass gap and a series of continuous 


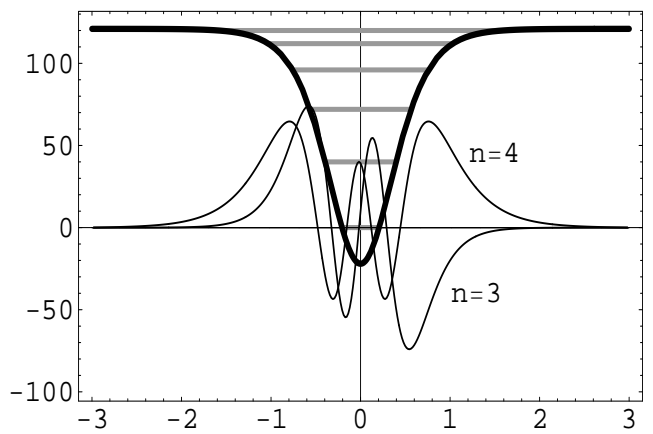

(a) Left chiral fermions, $a=0$

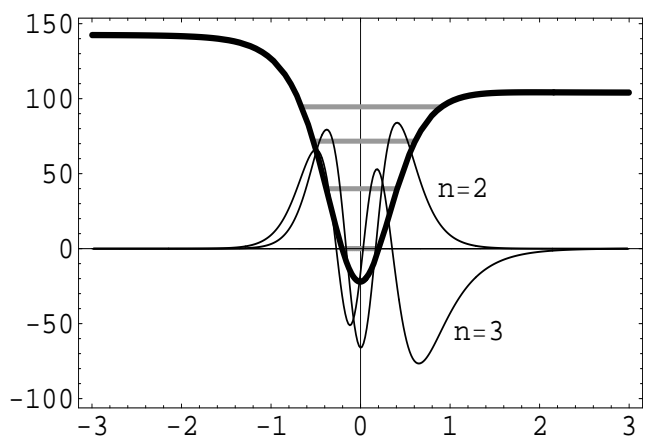

(c) Left chiral fermions, $a=0.1$

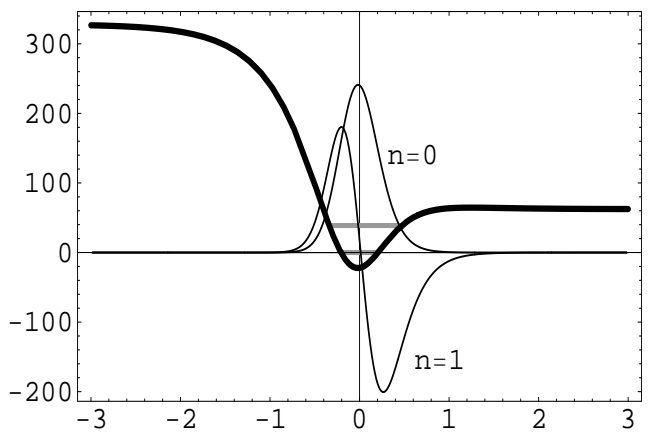

(e) Left chiral fermions, $a=0.5$

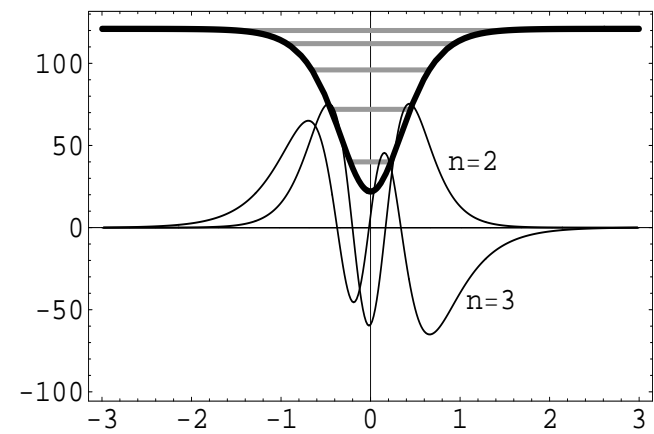

(b) Right chiral fermions, $a=0$

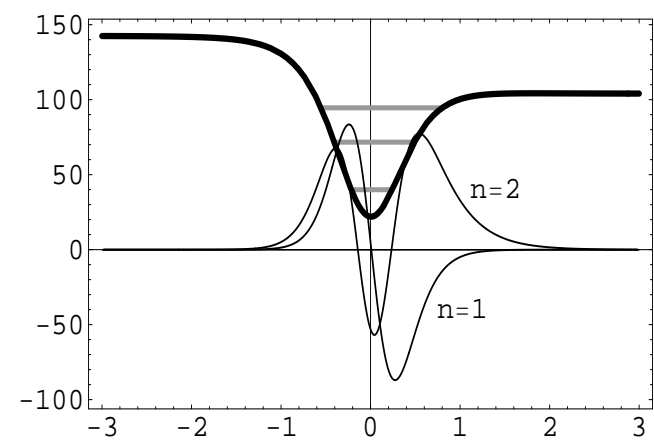

(d) Right chiral fermions, $a=0.1$

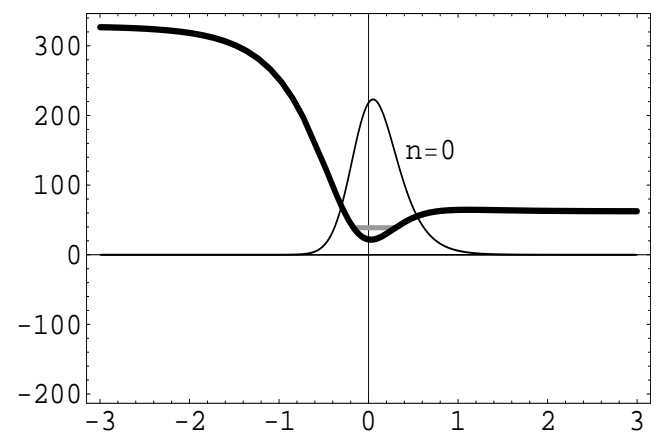

(f) Right chiral fermions, $a=0.5$

Figure 13: The potentials $V_{L, R}^{A}(z)$ (black thick lines), the mass spectrum $\mu_{L, R}^{2}$ (thick gray lines) and some eigenfunctions (black thin lines) for asymmetric $d S$ brane with $\beta=1, \delta=1 / 2, \eta=11$ and different $a$.

spectrum starting at positive $\mu^{2}$. It can be shown that the existence of such a mass gap is universal for all such $d S$ branes.

For the symmetric $d S$ brane, the spectrum of scalar KK modes consists of a zero mode 
and a set of continuous modes, i.e., there is only one bound mode (the zero mode). The massless mode is separated by a mass gap from the continuous modes. For the asymmetric $d S$ brane with a small asymmetric factor, the spectrum is same as the symmetric case. However, for a large enough asymmetric factor, the spectrum of scalar KK modes contains a bound massive KK mode besides a zero mode and a set of continuous modes, namely, there are two bound modes. For spin 1 vectors, the spectra of KK modes on both $d S$ branes are made up of a bound zero mode and a set of continuous ones. The asymmetric factor does not change the number of the vector bound modes.

It is shown that, without scalar-fermion coupling, there is no bound state for both the left and right chiral fermions. Hence, in order to localize the massless and massive left or right chiral fermions on the branes, some kind of kink-fermion coupling should be introduced. As examples, two types of kink-fermion couplings are investigated in detail. These situations can be compared with the case of the domain wall in the RS framework [20], where for localization of spin 1/2 field additional localization method by Jackiw and Rebbi [40] was introduced.

For the usual Yukawa coupling $\eta \bar{\Psi} \phi \Psi$, the potential for only one of the left and right chiral fermions has a finite well at the location of the brane and a finite barrier at each side which vanishes asymptotically. It is shown that there is only one single bound state (zero mode) which is just the lowest energy eigenfunction of the Schrödinger equation for the corresponding chiral fermions. Since the potentials for both left and right chiral fermions vanish asymptotically when far away from the brane, all values of $\mu^{2}>0$ are allowed, and there exists no mass gap but a continuous gapless spectrum of KK states with $\mu^{2}>0$. The massive KK modes asymptotically turn into continuous plane waves when far away from the brane [5, 10], which represent delocalized massive KK fermions.

For the scalar-fermion coupling $\eta \bar{\Psi} \sin \left(\frac{\phi}{\phi_{0}}\right) \cos ^{-\delta}\left(\frac{\phi}{\phi_{0}}\right) \Psi$ with positive $\eta$, the potential for the left chiral fermions has a finite well at the location of the brane, and a finite positive barrier at each side, which does not vanishes when far away from the brane. The potential is the modified Pöschl-Teller potential and suggest that there exist some discrete KK modes and a series of continuous ones. The discrete modes are bound states while the continuous ones are not. The total number of bound states is determined by four parameters: $\delta, \beta$, $a$ and $\eta$. The number of bound states of right chiral fermions is one less than that of left ones. The number of the bound states increases with the coupling constant $\eta$. For the case of the symmetric $d S$ brane, if $0<\eta<\beta / \delta$, there is only one left chiral fermion bound state which is just the left chiral fermion zero mode; if $\eta>\beta / \delta$, there are $N_{L}\left(N_{L} \geq 2\right)$ left chiral fermion bound states (including zero mode and massive KK modes) and $N_{L}-1$ right chiral fermion bound states (including only massive KK modes). For the asymmetric $d S$ brane scenario, the asymmetric factor $a$ reduces the number of the bound fermion KK modes. For large enough $a$, there would not be any right chiral fermion bound mode, but at least one left chiral fermion bound mode, i.e., the zero mode.

For fermions, localization property is decided by the coupling of fermion and scalar. For the first type of Yukawa coupling, $F(\phi(z)) \sim \arctan (\sinh z)$ is a usual kink which is almost a constant at large $z$. For the second type of coupling, $F(\phi(z))$ is another kink likes $\sinh z$, which increases quickly with $z$. In short, at large $z$, the first coupling is invariant, 
but the second one becomes stronger. Hence, the two different types of Yukawa couplings give different localization properties for fermions.

Finally, we give some brief discussion about graviton and gravitino localization on the studied branes. The Schrödinger potentials of graviton and gravitino KK modes are the same as that of scalar and fermion, respectively. Thus, for the symmetric $d S$ brane and the asymmetric $d S$ brane with small asymmetric factor, the spectrum of graviton KK modes consists of a discrete zero mode and a set of continuous modes. While for a large enough asymmetric factor, the spectrum of graviton KK modes contains a bound massive KK mode besides a zero mode and a set of continuous modes. The spectrum of gravitino is similar to that of fermion.

\section{Acknowledgement}

The authors are really grateful to the referee for his/her constructive comments and suggestions which considerably improved the paper. This work was supported by the National Natural Science Foundation of China (No. 10705013), the Doctor Education Fund of Educational Department of China (No. 20070730055) and the Fundamental Research Fund for Physics and Mathematics of Lanzhou University (No. Lzu07002).

\section{References}

[1] V.A. Rubakov and M.E. Shaposhnikov, Do we live inside a domain wall?, Phys. Lett. B $\mathbf{1 2 5}$ (1983) 136; V.A. Rubakov and M.E. Shaposhnikov, Extra space-time dimensions: towards a solution to the cosmological constant problem, Phys. Lett. B 125 (1983) 139; E.J. Squires, Dimensional reduction caused by a cosmological constant, Phys. Lett. B 167 (1986) 286;

K. Akama, Pregeometry, in Proceedings of the "Symposium on Gauge Theory and Gravitation", Nara Japan August 20-24 1982, K. Kikkawa, N. Nakanishi and H. Nariai eds., Springer-Verlag, Germany (1983), page 267-271 [Lect. Notes Phys. 176 (1982) 267], arXiv:hep-th/0001113.

[2] M. Visser, An exotic class of Kaluza-Klein models, Phys. Lett. B 159 (1985) 22, arXiv:hep-th/9910093.

[3] S. Randjbar-Daemi and C. Wetterich, Kaluza-Klein solutions with noncompact internal spaces, Phys. Lett. B 166 (1986) 65.

[4] L. Randall and R. Sundrum, A Large Mass Hierarchy from a Small Extra Dimension, Phys. Rev. Lett. 83 (1999) 3370 arxiv:hep-ph/9905221; An alternative to compactification, Phys. Rev. Lett. 83 (1999) 4690, arXiv:hep-th/9906064.

[5] J. Lykken and L. Randall, The Shape of Gravity, JHEP 0006 (2000) 014, arXiv:hep-th/9908076.

[6] I. Antoniadis, A possible new dimension at a few Tev, Phys. Lett. B 246 (1990) 377.

[7] N. Arkani-Hamed, S. Dimopoulos and G. Dvali, The hierarchy problem and new dimensions at a millimeter, Phys. Lett. B 429 (1998) 263, arXiv:hep-ph/9803315; I. Antoniadis, N. Arkani-Hamed, S. Dimopoulos and G. Dvali, New dimensions at a millimeter to a Fermi and superstrings at a TeV, Phys. Lett. B 436 (1998) 257, arXiv:hep-ph/9804398. 
[8] N. Arkani-Hamed, S. Dimopoulos, N. Kaloper and R. Sundtrum, Nucl. Phys. B 480 (2000) 193; S. Kachru, M. Schulz and E. Silverstein, Phys. Rev. D 62 (2000) 045021; A. Kehagias, A conical tear drop as a vacuum-energy drain for the solution of the cosmological constant problem, Phys. Lett. B 600 (2004) 133, arXiv:hep-th/0406025.

[9] G.D. Starkman, D. Stojkovic and M. Trodden, Large Extra Dimensions and Cosmological Problems, Phys. Rev. D 63 (2001) 103511, arXiv:hep-th/0012226; Homogeneity Flatness and Large Extra Dimensions, Phys. Rev. Lett. 87 (2001) 231303, arXiv:hep-th/0106143.

[10] O. DeWolfe, D.Z. Freedman, S.S. Gubser and A. Karch, Modeling the fifth dimension with scalars and gravity, Phys. Rev. D 62 (2000) 046008, arXiv:hep-th/9909134.

[11] M. Gremm, Four-dimensional gravity on a thick domain wall, Phys. Lett. B 478 (2000) 434, arXiv:hep-th/9912060; Thick domain walls and singular spaces, Phys. Rev. D 62 (2000) 044017, arXiv:hep-th/0002040; K. Ghoroku and M. Yahiro, Instability of thick brane worlds, hep-th/0305150; A. Kehagias and K. Tamvakis, A Self-Tuning Solution of the Cosmological Constant Problem, Mod. Phys. Lett. A 17 (2002) 1767, arXiv:hep-th/0011006; Localized Gravitons, Gauge Bosons and Chiral Fermions in Smooth Spaces Generated by a Bounce, Phys. Lett. B 504(2001) 38, arXiv:hep-th/0010112; M. Giovannini, Gauge-invariant fluctuations of scalar branes, Phys. Rev. D 64 (2001) 064023, arXiv:hep-th/0106041; Localization of metric fluctuations on scalar branes, Phys. Rev. D 65 (2002) 064008, arXiv:hep-th/0106131; S. Kobayashi, K. Koyama and J. Soda, Thick brane worlds and their stability, Phys. Rev. D 65 (2002) 064014; R. Gregory and A. Padilla, Nested braneworlds and strong brane gravity, Phys. Rev. D 65 (2002) 084013, arXiv:hep-th/0104262.

[12] C. Csaki, J. Erlich, T. Hollowood and Y. Shirman, Universal Aspects of gravity localized on thick branes, Nucl. Phys. B 581 (2000) 309, arXiv:hep-th/0001033.

[13] A. Campos, Critical phenomena of thick brane in warped space-time, Phys. Rev. Lett. 88 (2002) 141602, arXiv:hep-th/0111207.

[14] A. Z. Wang, Thick de Sitter brane worlds, dynamic black holes and localization of gravity, Phys. Rev. D 66 (2002) 024024, arXiv:hep-th/0201051.

[15] R. Emparan, R. Gregory and C. Santos, Black holes on thick branes, Phys. Rev. D 63 (2001) 104022; A. Melfo, N. Pantoja and A. Skirzewski, Thick domain wall space-time with and without reflection symmetry, Phys. Rev. D 67 (2003) 105003, arXiv:gr-qc/0211081. K.A. Bronnikov and B.E. Meierovich, A general thick brane supported by a scalar field, Grav. Cosmol. 9 (2003) 313; O. Castillo-Felisola, A. Melfo, N. Pantoja and A. Ramirez, Localizing gravity on exotic thick three-branes, Phys. Rev. D 70 (2004) 104029; D. Bazeia, L. Losano and R. Menezes, First-order framework and generalized global defect solutions, Phys. Lett. B 668 (2008) 246, arXiv:0807.0213[hep-th]; D. Bazeia, A.R. Gomes, L. Losano and R. Menezes, Braneworld Models of Scalar Fields with Generalized Dynamics, Phys. Lett. B 671 (2009) 402, arXiv:0808.1815[hep-th]; R. Koley, J. Mitra and S. SenGupta, Modulus stabilization of generalized Randall Sundrum model with bulk scalar field, to appear in Euro. Phys. Lett., arXiv:0809.4102[hep-th].

[16] V. Dzhunushaliev, V. Folomeev, D. Singleton and S. Aguilar-Rudametkin, 6D thick branes from interacting scalar fields, Phys. Rev. D 77 (2008) 044006, arXiv:hep-th/0703043; V. Dzhunushaliev, V. Folomeev, K. Myrzakulov and R. Myrzakulov, Thick brane in $7 D$ and $8 D$ spacetimes, Gen. Rel. Grav. 41 (2009) 131, arXiv:0705.4014; D. Bazeia, F.A. Brito and J.R. Nascimento, Supergravity brane worlds and tachyon potentials, Phys. Rev. D 68 (2003) 085007, arXiv:hep-th/0306284; D. Bazeia, F.A. Brito and A.R. Gomes, Locally Localized 
Gravity and Geometric Transitions, JHEP 0411 (2004) 070, arXiv:hep-th/0411088; D. Bazeia and A.R. Gomes, Bloch Brane, JHEP 0405 (2004) 012, arXiv:hep-th/0403141; D. Bazeia, F.A. Brito and L. Losano, Scalar fields, bent branes, and $R G$ flow, JHEP 0611 (2006) 064, arXiv:hep-th/0610233; D. Bazeia, A.R. Gomes and L. Losano, Gravity localization on thick branes: a numerical approach, arXiv:0708.3530[hep-th]; D. Bazeia, C. Furtado and A.R. Gomes, Brane structure from scalar field in warped spacetime, JCAP 0402 (2004) 002, arXiv:hep-th/0308034.

[17] M. Minamitsuji, W. Naylor and M. Sasaki, Quantum fluctuations on a thick de Sitter brane, Nucl. Phys. B 737 (2006) 121, arXiv:hep-th/0508093.

[18] N. Sasakura, JHEP 0202 (2002) 026, arXiv:hep-th/0201130; I. Olasagasti and K. Tamvakis, Gravity in higer codimension de Sitter Brane Worlds, Phys. Rev. D 68 (2003) 064016, arXiv:hep-th/0303096. V. Dzhunushaliev, V. Folomeev and M. Minamitsuji, Thick de Sitter brane solutions in higher dimensions, Phys. Rev. D 79 (2009) 024001, arXiv:0809.4076.

[19] R. Guerrero, R.O. Rodriguez, R. Ortiz and R. Torrealba, De Sitter and double irregular domain walls, Gen. Rel. Grav. 38 (2006) 845, arXiv:gr-qc/0504080; R. Guerrero, R. O. Rodriguez and R. Torrealba, De-Sitter and double asymmetric brane worlds, Phys. Rev. D 72 (2005) 124012, arXiv:hep-th/0510023v2.

[20] B. Bajc and G. Gabadadze, Localization of matter and cosmological constant on a brane in anti de Sitter space, Phys. Lett. B 474 (2000) 282, arXiv:hep-th/9912232.

[21] I. Oda, Localization of matters on a string-like defect, Phys. Lett. B 496 (2000) 113, arXiv:hep-th/0006203.

[22] Y.-X. Liu, X.-H. Zhang, L.-D. Zhang and Y.-S. Duan, Localization of Matters on Pure Geometrical Thick Branes, JHEP 0802 (2008) 067, arXiv:0708.0065[hep-th].

[23] Y. Grossman and N. Neubert, Neutrino masses and mixings in non-factorizable geometry, Phys. Lett. B 474 (2000) 361, arXiv:hep-ph/9912408; R. Koley and S. Kar, A novel braneworld model with a bulk scalar field, Phys. Lett. B 623 (2005) 244, arXiv:hep-th/0507277; [Erratum ibid. 631 (2005) 199; A. Melfo, N. Pantoja and J.D. Tempo, Fermion localization on thick branes, Phys. Rev. D 73 (2006) 044033, arXiv:hep-th/0601161; R. Koley, J. Mitra and S. SenGupta, Chiral fermions in a spacetime with multiple warping, Phys. Rev. D 78 (2008) 045005, arXiv:0804.1019[hep-th]; R. Koley, J. Mitra and S. SenGupta, Fermions in branes with nonzero cosmological constant: a new anthropic principle, arXiv:0806.0455[hep-ph]; R. Koley, Localization of fields on brane, in Proceedings of the Workshop on "Physics of warped extra dimensions", Kharagpur India February 21-23 2008, Page 19-25, arXiv:0812.1423[hep-th].

[24] T.R. Slatyer and R.R. Volkas, Cosmology and fermion confinement in a scalar-field generated domain wall brane in five dimensions, JHEP 0704 (2007) 062, arXiv:hep-ph/0609003; R. Davies, D.P. George and R.R. Volkas, Standard model on a domain wall brane, Phys. Rev. D 77 (2008) 124038, arXiv:0705.1584[hep-ph].

[25] S. Ichinose, Fermions in Kaluza-Klein and Randall-Sundrum theories, Phys. Rev. D 66 (2002) 104015, arXiv:hep-th/0206187.

[26] C. Ringeval, P. Peter and J.P. Uzan, Localization of massive fermions on the brane, Phys. Rev. D 65 (2002) 044016, arXiv:hep-th/0109194.

[27] T. Gherghetta and M. Shaposhnikov, Localizing gravity on a string-like defect in six dimensions, Phys. Rev. Lett. 85 (2000) 240, arXiv:hep-th/0004014. 
[28] I.P. Neupane, Consistency of higher derivative gravity in the brane background, JHEP 0009 (2000) 040, arXiv:hep-th/0008190; I.P. Neupane, Completely localized gravity with higher curvature terms, Class. Quant. Grav. 19 (2002) 5507, arXiv:hep-th/0106100.

[29] S. Randjbar-Daemi and M. Shaposhnikov, Fermion zero-modes on brane-worlds, Phys. Lett. B 492 (2000) 361, arXiv:hep-th/0008079.

[30] R. Koley and S. Kar, Scalar kinks and fermion localisation in warped spacetimes, Class. Quant. Grav. 22 (2005) 753, arXiv:hep-th/0407158.

[31] S.L. Dubovsky, V.A. Rubakov and P.G. Tinyakov, Brane world: disappearing massive matter, Phys. Rev. D 62 (2000) 105011, arXiv:hep-th/0006046.

[32] Y. Brihaye and T. Delsate, Remarks on bell-shaped lumps: stability and fermionic modes, Phys. Rev. D 78 (2008) 025014, arXiv:0803.1458[hep-th].

[33] O. Arias, R. Cardenas and I. Quiros, Thick Brane Worlds Arising From Pure Geometry, Nucl. Phys. B 643 (2002) 187, arXiv:hep-th/0202130. N. Barbosa-Cendejas and A. Herrera-Aguilar, $4 D$ gravity localized in non $Z_{2}$-symmetric thick branes JHEP 0510 (2005) 101, arXiv:hep-th/0511050. N. Barbosa-Cendejas and A. Herrera-Aguilar, Localization of $4 D$ gravity on pure geometrical thick branes, Phys. Rev. D 73 (2006) 084022, arXiv:hep-th/0603184.

[34] N. Barbosa-Cendejas, A. Herrera-Aguilar, M. A. Reyes and C. Schubert, Mass gap for gravity localized on Weyl thick branes, Phys. Rev. D 77 (2008) 126013, arXiv:0709.3552[hep-th]; N. Barbosa-Cendejas, A. Herrera-Aguilar, U. Nucamendi and I. Quiros, Mass hierarchy and mass gap on thick branes with Poincare symmetry, arXiv:0712.3098[hep-th].

[35] Y.-X. Liu, L.-D. Zhang, S.-W. Wei and Y.-S. Duan, Localization and Mass Spectrum of Matters on Weyl Thick Branes, JHEP 0808 (2008) 041, arXiv:0803.0098[hep-th].

[36] G. Goetz, J. Math. Phys. 31 (1990) 2683.

[37] R. Gass and M. Mukherjee, Domain wall spacetimes and particle motion, Phys. Rev. D 60 (1999) 065011, arXiv:gr-qc/9903012.

[38] R. Guerrero, A. Melfo and N. Pantoja, Self-gravitating domain walls and the thin-wall limit, Phys. Rev. D 65 (2002) 125010, arXiv:gr-qc/0202011.

[39] G. Dvali and M. Shifman, Domain walls in strongly coupled theories, Phys. Lett. B 396 (1997) 64, arxiv:hep-th/9612128.

[40] R. Jackiw and C. Rebbi, Solitons with fermion number 1/2, Phys. Rev. D 13 (1976) 3398.

[41] Y.-X. Liu, L.-D. Zhang, L.-J. Zhang and Y.-S. Duan, Fermions on Thick Branes in the Background of Sine-Gordon Kinks, Phys. Rev. D 78, 065025 (2008), arXiv:0804.4553[hep-th].

[42] S. Ichinose, Some Properties of Domain Wall Solution in the Randall-Sundrum Model, Phys. Rev. D 65 (2002) 084038; Class. Quant. Grav. 18 (2001) 5239.

[43] Y.-X. Liu, L. Zhao and Y.-S. Duan, Localization of Fermions on a String-like Defect, JHEP 0704 (2007) 097, arXiv:hep-th/0701010.

[44] G. de Pol, H. Singh and M. Tonin, Action with manifest duality for maximally supersymmetric six-dimensional supergravity, Int. J. Mod. Phys. A 15 (2000) 4447, arXiv:hep-th/0003106. 
[45] S.L. Parameswaran, S. Randjbar-Daemi and A. Salvio, Gauge Fields, Fermions and Mass Gaps in 6D Brane Worlds, Nucl. Phys. B 767 (2007) 54, arXiv:hep-th/0608074.

[46] Y.-X. Liu, L. Zhao, X.-H. Zhang and Y.-S. Duan, Fermions in Self-dual Vortex Background on a String-like Defect, Nucl. Phys. B 785 (2007) 234, arXiv:0704.2812[hep-th].

[47] Y.-Q. Wang, T.-Y. Si, Y.-X. Liu and Y.-S. Duan, Fermionic zero modes in self-dual vortex background, Mod. Phys. Lett. A 20 (2005) 3045, arXiv:hep-th/0508111; Y.-S. Duan, Y.-X. Liu and Y.-Q. Wang, Fermionic Zero Modes in Gauge and Gravity Backgrounds on T ${ }^{2}$, Mod. Phys. Lett. A 21 (2006) 2019, arXiv:hep-th/0602157; Y.X. Liu, Y.Q. Wang and Y.S. Duan, Fermionic zero modes in self-dual vortex background on a torus, Commun. Theor. Phys. 48 (2007) 675.

[48] S. Rafael and S. Torrealba, Exact Abelian Higgs Vortices as 6D Brane Worlds, arXiv:0803.0313[hep-th].

[49] G. Starkman, D. Stojkovic and T. Vachaspati, Zero modes of fermions with a general mass matrix, Phys. Rev. D 65 (2002) 065003; Neutrino zero modes on electroweak strings, Phys. Rev. D 63 (2001) 085011; D. Stojkovic, Fermionic zero modes on domain walls, Phys. Rev. D 63 (2000) 025010.

[50] R. Jackiw and P. Rossi, Zero modes of the vortex-fermion system, Nucl. Phys. B 190 (1981) 681. 